ISSN: 0212-5374

DOI: http://dx.doi.org/10.14201/et2014321147176

\title{
INCIDENCIA DE LOS HÁBITOS EDUCATIVOS EN EL APRENDIZAJE DEL ALUMNADO DE EDUCACIÓN INFANTIL
}

\author{
Incidence of the educational habits in the learning \\ of students of preschool education
}

\section{Incidence des habitudes éducatifs sur l'apprentissage des élèves d'Éducation Maternelle}

\author{
María MARTínez Redondo*, Miguel PéRez FerRA*** y Beatriz SierRA \\ Y ARIZMENDIARRIETA**** \\ *IES “Veracruz”. Correo-e: mredondo@ujaen.es \\ ** Universidad de Jaén. Correo-e: mperez@ujaen.es \\ **** Universidad de Oviedo. Correo-e: bsierra@uniovi.es
}

Recibido: 03.10.2013; Aceptado: 18.12.2013; Publicado: 31.10.2014

BIBLID [0212-5374 (2014) 32, 2; 147-176]

Ref. Bibl. MARÍA MARTÍNEZ REDONDO, MIGUEL PÉREZ FERRA y BEATRIZ SIERRA Y ARIZMENDIARRIETA. Incidencia de los hábitos educativos en el aprendizaje del alumnado de Educación Infantil. Enseñanza \& Teaching, 32, 2-2014, 147-176.

RESUMEN: La etapa de Educación Infantil es fundamental para el posterior desarrollo del alumnado. Se debe garantizar un entorno de aprendizaje basado en actividades lúdicas y significativas que permita a los niños y niñas crecer en unas condiciones apropiadas para el desarrollo de hábitos educativos que figuran en el currículum de Educación Infantil. El profesorado debe dirigir su metodología a la consolidación y afianzamiento de hábitos educativos tales como deportivos y tecnológicos, de lectura y escritura, morales y conductuales, ambientales, de higiene y autonomía personal. 
MARÍA MARTÍNEZ REDONDO, MIGUEL PÉREZ FERRA Y BEATRIZ SIERRA Y ARIZMENDIARRIETA INCIDENCIA DE LOS HÁBITOS EDUCATIVOS EN EL APRENDIZAJE DEL ALUMNADO DE EDUCACIÓN INFANTIL

En este artículo, presentamos los resultados de un trabajo de investigación en el que se recogen, a través de una metodología cuantitativa, las opiniones del profesorado de Educación Infantil de la zona de Sierra de Segura, El Condado y Las Villas, cuya cabecera de comarca es úbeda, provincia de Jaén, respecto a qué tipología de hábitos educativos es más recomendable aplicar en la práctica educativa según las directrices que nos brinda el currículum de Educación Infantil.

Los resultados evidencian, según las opiniones mostradas por el profesorado de Educación Infantil, la incipiente necesidad de basar la práctica docente en la trasmisión y desarrollo de hábitos educativos en el alumnado de tres a seis años, generando nuevos planteamientos, conocimientos, habilidades y actitudes imprescindibles para la futura formación del alumnado.

Palabras clave: Educación Infantil, hábitos educativos, aprendizaje, proceso educativo, currículum.

SUMMARY: The preschool (ages 3-6) is key for the future development the students in the society that surrounds them. It must guarantee an environment of learning based on playful and significant activities that allows the children to grow in preschool appropriate for the development of educational habits that appear in the curriculum. Teacher staff must direct their methodology to consolidate and backing of such educational habits as sports and technology, of reading and writing, moral and behavioral, environment, of hygiene and personal autonomy.

In this article we show the results of a work of our research in which gathered, information across a quantitative methodology. The opinions of the teachers of preschool of the area of Sierra de Segura, El Condado y Las Villas, where Úbeda is the head of the province of Jaén with regard to the type of educational habits that are more advisable to apply in the educational practice depending on the directives that preschool curriculum offers to us.

The results demonstrate, according to the opinions showed preschool teachers, that the recent teachers need to base their methods in the transmission and development of 3-6 years olds education, generating new approaches, knowledge, skills and indispensable disposition for their future formation.

Key words: preschool, education habits, learning, curriculum, educational process.

RÉSUMÉ: L'étape d'Éducation Maternelle est décisive pour le plein développement des élèves dans la société qui les entoure. Elle doit garantir un environnement d'apprentissage basé sur des activités ludiques et significatives qui permetten aux jeunes élèves de grandir dans des conditions appropriées pour le développment d'habitudes éducatifs qui figurent dans le curriculum d'Éducation Maternelle. Le professorat doit diriger sa méthodologie vers la consolidation et la garantie d'habitudes éducatifs tels que sportifs et technologiques, d'une lecture et une écriture, et moraux et conductuales, environnementaux, une hygiène et une autonomie personnelle. 
MARÍA MARTÍNEZ REDONDO, MIGUEL PÉREZ FERRA Y BEATRIZ SIERRA Y ARIZMENDIARRIETA INCIDENCIA DE LOS HÁBITOS EDUCATIVOS EN EL APRENDIZAJE DEL ALUMNADO DE EDUCACIÓN INFANTIL

Dans cet article nous présentons les résultats d'un travail de recherche dans lequel sont recuillis, avec une méthodologie quantitative, les opinions du professorat d'Éducation Maternelle de la zone de Sierra de Segura, El Condado et Las Villas dont la ville principale est Úbeda (Jaén), par rapport à la typologie d'habitudes éducatifs la plus recommandable d'appliquer en pratique éducative selon les directrices que le curriculum de l'Éducation Maternelle nous offre.

À partir des opinions montrées par le professorat d'Éducation Maternelle, les résultats rendrent évidents, le besoin naissant de baser la pratique enseignante sur la transmission et le développement d'habitudes éducatifs sur l'ensemble des élèves de trois à six ans, en générant de nouveaux développements, connaissances, habiletés et attitudes indispensables pour la éducation de l'alumnat.

Mots clés: Éducation Maternelle, habitudes éducatifs, apprentissage, processus éducatif, curriculum.

\section{APROXIMACIÓN AL TEMA OBJETO DE ESTUDIO}

El término "hábito" etimológicamente procede del latín "habitus" y "es el modo especial de proceder o conducirse, adquirido por repetición de actos iguales o semejantes u originado por tendencias instintivas" (RAE, 2006: 756).

Iniciamos esta justificación mediante una aproximación genealógica, cuya revisión de la literatura conduce a analizar corrientes, autores y aspectos legislativos de la Educación Infantil; donde la investigación psicoeducativa manifiesta la importancia que tienen los hábitos en esta etapa educativa, afirmación ya corroborada en la década de los años noventa por los estudios de Dahlberg y Asen (1994); Moss (1994); Gútiez (1995); Moss y Penn (1996) y permite determinar cuál es su situación en cada momento, establecida por la realidad de la etapa educativa y la situación particular de cada momento cultural en el que se valora.

El análisis histórico es fundamental para la justificación en ciencias sociales, ya que permite determinar lo que cada cultura, en cada momento, aporta a una realidad que evoluciona longitudinalmente y está mediada por las condiciones del entorno en ese tiempo.

El origen de la Educación Infantil se sitúa en el siglo XIX y está estrechamente ligado a Friedrich Fröebel. Su concepción de la enseñanza priorizaba la exigencia de los niños como responsables de su proceso de aprendizaje. Aplica una metodología eminentemente lúdica, propiciando aprendizajes significativos basados en el juego. En este mismo sentido se puede hablar de Joham H. Pestalozzi, que impulsó la formación específica de profesoras para que se dedicaran a enseñar a los niños en los Kindergarten. En España se ha de hacer especial mención a Pablo Montesinos, que formó al profesorado encargado de instruir a estas edades. En el primer tercio del siglo xx se amplió el campo educativo con la creación de instituciones educativas, tanto públicas como privadas, para atender al alumnado de Educación Infantil, acciones impulsadas fundamentalmente por Montessori y Decroly. 

INCIDENCIA DE LOS HÁBITOS EDUCATIVOS EN EL APRENDIZAJE DEL ALUMNADO DE EDUCACIÓN INFANTIL

El pensamiento de ambos ha sido el eje vertebrador de lo que hoy entendemos por Educación Infantil.

En el ámbito español, el comienzo de la dictadura en el año 39 ralentizó el desarrollo de la etapa referenciada, de modo que pierde identidad propia y gana sentido propedéutico, considerándola de preparación hacia la Educación Primaria. Solo a partir de 1970, con la puesta en marcha de la Ley 14/1970, de 4 de agosto, General de Educación (LGE) y Financiamiento de la Reforma Educativa, se aprecia un cambio favorable hacia la mejora de los estudios de Educación Infantil.

Es a partir de la década de los años setenta cuando España experimenta un avance en el ámbito pedagógico, concretamente, la LGE de 1970 dedica un espacio a la etapa de Educación Infantil, como periodo con identidad propia, perdiendo el marcado carácter asistencial de tiempo atrás. Dicho avance se va consolidando en sucesivas reformas educativas: LOGSE (Ley Orgánica General del Sistema Educativo), LOCE (Ley Orgánica de Calidad de la Educación) y LOE (Ley Orgánica de Educación), avances que quedan expuestos en la Tabla 1.

La Ley General de Educación (LGE) propuso cambios relevantes para la educación infantil, dotando la etapa de recursos y métodos eficaces. La LOGSE planteó una ambiciosa propuesta pedagógica para la etapa de Educación Infantil, que aportaba autonomía respecto a otras etapas educativas. Promovió un sistema de enseñanza constructivista propiciando un currículum abierto y flexible, favoreciendo el desarrollo del alumnado en las áreas de identidad y autonomía personal, medio físico y social, comunicación y representación.

La etapa se ofertaba con carácter gratuito aunque no obligatorio, comprendiendo dos ciclos escolares, de cero a tres años y de tres a seis años respectivamente. Sustituyó el término educación preescolar de la LGE, por el de Educación Infantil. La LOCE no se implantó, quedando derogada el 24 de mayo de 2006 por la LOE, razón por la que sus propuestas no se llevaron a la práctica; sin embargo, entre ellas hay que señalar que el primer tramo de la Educación Infantil recobró su carácter asistencial. La LOE no introdujo cambios profundos con respecto a las normativas citadas, siendo un compendio de la LOGSE y la LOCE. La etapa de Educación Infantil pierde el carácter propedéutico y va consolidando su entidad propia como etapa educativa. En definitiva, recuperó su identidad educativa, sin realizar diferenciación por ciclos.

Coincidimos con Zabalza (1996) en que la Escuela Infantil es:

El marco institucional que suministra al niño una amplia gama de estímulos y oportunidades polivalentes, una institución formativa e intencionalmente organizada como una red de aprendizajes cognitivos-intelectuales, ético-sociales y físico-expresivos, para que el niño se acerque a su techo potencial, es decir, a lo que la pedagogía ha definido siempre como el máximo desarrollo profesional y aptitudinal de cada persona (pp. 59-74). 
Las reflexiones precedentes ponen de manifiesto la consideración de la Educación Infantil como una etapa en la que se inicia el desarrollo de la "integridad" e "integralidad" del niño o niña como ser humano y se le hace partícipe y protagonista de su propia formación a través del descubrimiento de la realidad que le circunda.

\section{NATURALEZA Y NECESIDAD DE LOS HÁBITOS EDUCATIVOS EN EDUCACIÓN INFANTIL}

El tratamiento didáctico de los hábitos educativos está determinado por los estilos de aprendizaje que utilicemos. Consideramos interesante el posicionamiento respecto a qué es un hábito, ya que permite plantear finalidades y seleccionar pautas de actuación docentes.

Se parte de la idea de que un hábito es un proceso que demanda cierto tiempo para su asimilación y habilitación, que facilita el desarrollo personal, apoyándose en adquisiciones progresivas que permiten superar el determinismo biológico y la dependencia respecto a otras personas en la satisfacción de necesidades.

En Educación Infantil se puede hablar de cinco grandes tipos de hábitos, a saber: morales, conductuales y sociales; ambientales; hábitos de lectura y escritura; hábitos tecnológicos y deportivos, y hábitos de higiene y autonomía personal, que quedan reflejados en la Figura 1.

FIGURA 1

Hábitos incluidos en el currículum de Educación Infantil

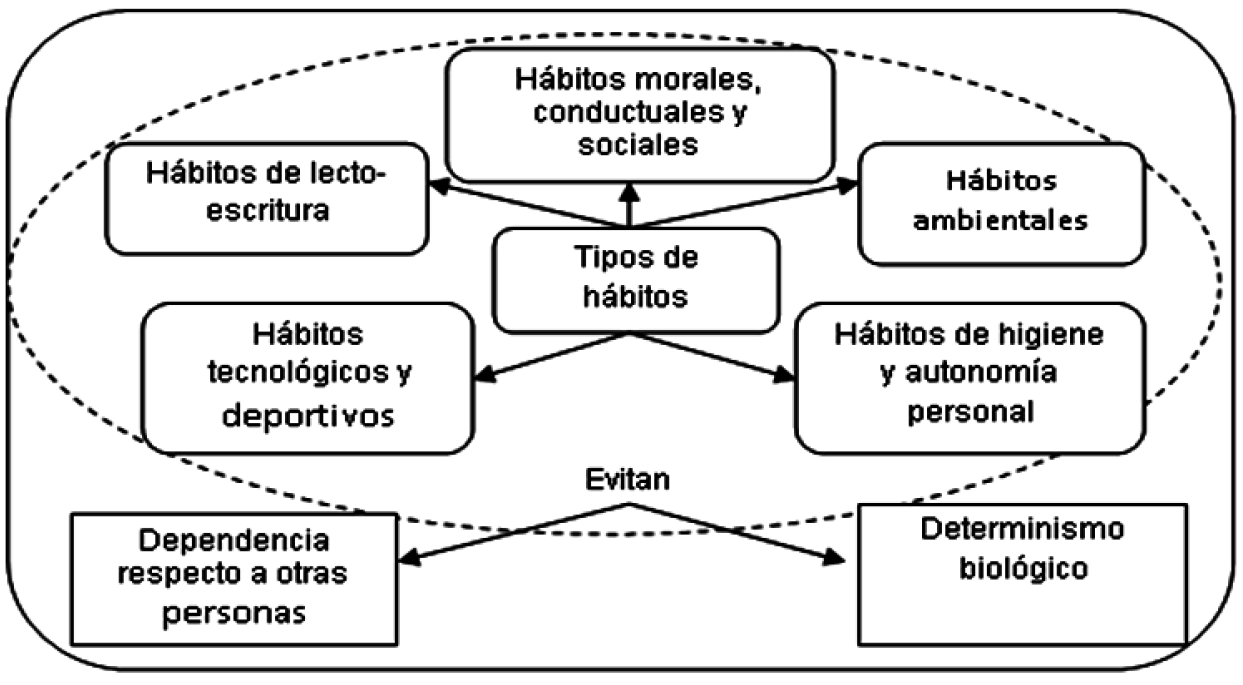



INCIDENCIA DE LOS HÁBITOS EDUCATIVOS EN EL APRENDIZAJE DEL ALUMNADO DE EDUCACIÓN INFANTIL

El trabajo con los hábitos supone una planificación en el ámbito de la programación didáctica, planificación que va secuenciada en orden a los tópicos que se siguen en las unidades didácticas y adecuadas a la madurez del niño y temática de las mismas. El desarrollo de los hábitos en el alumnado supone en el profesorado: observar, escuchar y acoger; además de proceder con una conducta coherente y estable (el profesorado es referente del alumnado). Pero el desarrollo de hábitos no se sustrae al principio de contextualización a la cultura, a la madurez y a los conocimientos previos que tiene el niño o la niña; por consiguiente, se parte de sus posibilidades y con disposición comprensiva por parte del profesorado. El respeto a la iniciativa del alumnado en sus actuaciones favorecerá su autoestima, reflexionando conjuntamente con él o ella sobre las posibles causas de los intentos fallidos; hará un uso continuado del lenguaje, con el fin de dar sentido y significado a lo que hace la niña o niño.

Enumerados los aspectos precedentes sobre nuestra concepción de hábito y planteados algunos principios de procedimiento para intervenir, es más que conveniente reflexionar sobre las aportaciones de la literatura en relación a los cinco tipos de hábitos que se pueden considerar claves en la Educación Infantil.

\subsection{Hábitos morales, conductuales y sociales}

El conjunto de pautas, gestos y conductas que se van haciendo permanentes con el paso del tiempo y que ayudan a delimitar los rasgos personales del alumnado constituyen la base en los hábitos morales, conductuales y sociales. Así pues, como docentes es necesario plantear situaciones lúdicas para aprender a respetar las diversas formas de opinión y pensamiento y resaltar la importancia de que todas las personas tienen los mismos derechos, generando las relaciones sociales basadas en el bienestar mutuo. Las investigaciones llevadas a cabo en este campo han puesto de manifiesto que los niños ya poseen a los tres años capacidad para distinguir las diferencias raciales y manifestar actitudes negativas o positivas, así como prejuicios hacia grupos en particular (Ayers, 2004; Connolly, 2003). Mundine y Giugni (2006) han manifestado que las disquisiciones aludidas líneas atrás como las actitudes mencionadas inciden en su comunicación con el medio social. Realidad que implica la imperiosa necesidad de crear situaciones sociales adecuadas que eliminen este tipo de prejuicios raciales, consolidando hábitos apropiados de interacción social desde el contexto escolar.

En el mismo sentido, percibimos el logro de actitudes beneficiosas para el alumnado de tres a seis años y su familia, implicando la práctica de habilidades sociales que favorezcan un sistema educativo exitoso y una vida feliz (Farrel, Tayler y Tennent, 2006). Las investigaciones aportadas por la literatura aconsejan que durante la etapa de Educación Infantil se deben potenciar: "las estrategias destinadas a crear condiciones de igualdad en los primeros años de vida para que sea posible alcanzar niveles dignos de equidad en la vida adulta" (Tedesco, 2010: 30). En conclusión: "es en la primera infancia donde las oportunidades sociales, económicas 
MARÍA MARTÍNEZ REDONDO, MIGUEL PÉREZ FERRA Y BEATRIZ SIERRA Y ARIZMENDIARRIETA INCIDENCIA DE LOS HÁBITOS EDUCATIVOS EN EL APRENDIZAJE DEL ALUMNADO DE EDUCACIÓN INFANTIL

y culturales nos igualan o diferencian" (Castañeda, 2010: 32). Así pues, se debe considerar la Educación Infantil como la etapa idónea para desarrollar hábitos que ayuden a conseguir un equilibrio emocional en los infantes.

\subsection{Apreciación y cuidado del entorno y del propio cuerpo}

Otro grupo de hábitos de capital importancia se concreta en la apreciación y cuidado del entorno y del propio cuerpo, fomentando una vida saludable y respetuosa con el medio ambiente. Esta nueva realidad está muy relacionada con los hábitos morales, de conducta y de interacción social, a fin de alcanzar decisiones unificadas y productivas (Foro Internacional ONG y MMSS, 1994). Son varios los autores que han elaborado actividades apropiadas para esta etapa, tales como: reciclaje, ayuda a buen desarrollo sostenible, creación de programas de recogida de residuos, planes medioambientales o difusión de programas para una alimentación saludable y cuidado del propio cuerpo, etc., en definitiva, se trata de desarrollar hábitos adecuados que fomenten la concienciación del niño por el cuidado del medio ambiente (Bohera et al., 2010). El desarrollo de este tipo de hábitos saludables y alimenticios permite un consumo responsable desde las edades más tempranas, aspectos tales como: mantener una alimentación equilibrada, reciclar, contribuir a evitar la contaminación acústica o utilizar productos reciclados, entre otros, los cuales son de vital importancia. Todos ellos, simultáneamente, constituyen estrategias básicas muy beneficiosas para el cuidado del medio ambiente (Estivill y Domènech, 2008). Ello será posible si se trabaja la educación ambiental en los primeros momentos de incorporación a la escuela, generando hábitos perdurables. Pero siendo la educación un proceso a lo largo de toda la vida (Otero y Bruno, 2009), estos hábitos "pueden seguir desarrollándose desde los temas transversales o en las áreas curriculares", así lo pone de manifiesto Perreoud (2012).

En la línea anteriormente descrita también se manifiestan autores como Freire (2011), que considera la etapa de Educación Infantil como el mejor momento para fomentar la interacción del alumnado con el medio físico. García (2009) concreta algo más y plantea la etapa de Educación Infantil como un periodo idóneo para adquirir hábitos relacionados con: "no tirar la basura", "usar el grifo adecuadamente", "reciclar", etc. A su vez, autores como Mendoza, Sagrera y Batista (1994); Rodrigo, Maíquez, García, Mendoza, Martínez y Martín (2004) aluden a los hábitos relacionados con la salud como imprescindibles para fomentar el equilibrio emocional y el desarrollo personal. En general, los hábitos aludidos favorecen la adopción de estilos de vida apropiados con el medio ambiente, donde el profesorado debe resaltar la importancia de llevar a cabo una alimentación rica, sana y equilibrada. Además, la propuesta de actividades de reflexión y de intercambio favorece la prevención de accidentes, los hábitos de salud y alimentación. También, y desde el aula, el profesorado debe propiciar aquellas actividades donde se potencie el cuidado y respeto al medio ambiente y al reciclaje de materiales en su actividad cotidiana. 

INCIDENCIA DE LOS HÁBITOS EDUCATIVOS EN EL APRENDIZAJE DEL ALUMNADO DE EDUCACIÓN INFANTIL

\subsection{Hábitos relacionados con la lectoescritura}

Si hay una realidad que pone en comunicación al niño con el mundo, favorece su socialización a través de la interiorización de la cultura de su entorno y le permite expresar lo que piensa y siente, esa realidad responde a los procesos lectoescritores. No es suficiente que el alumnado, por el hecho de estar presente en el lenguaje oral, pueda adquirir hábitos de lectura, pues: "resulta difícil demostrar que la formación de representaciones fonológicas se establezca por el mero hecho de estar expuestos a la lengua oral" (Jiménez y O'Shanahan, 2008: 4). Los escolares, además del reconocimiento visual de letras y palabras, y del desarrollo de la conciencia fonológica, necesitan para adquirir el hábito lector de otras habilidades cognoscitivas, tales como: transformación de grafemas en fonemas y viceversa, así se han manifestado autores como González, Romero y Blanca (1995); Gallego (2006) o Labat, Ecalle y Magnan (2010).

De igual modo, el profesorado debe priorizar la escritura como un aspecto de especial relevancia a través de cuentos, comparaciones entre letras-frases, copias, rótulos, revistas, etc., donde sea imprescindible aplicar estrategias educativas para fomentar tanto la lectura como la escritura. Se recomienda dedicar todos los días una parte de la jornada escolar al proceso lectoescritor. Para ello, existen programas de carácter perceptivo que ayudan a interiorizar las habilidades de lectura, velocidad, fluidez verbal y semántica (Soriano, 2007). Este tipo de programas son recomendables para afianzar, desde edades tempranas, hábitos que ayudan conseguir un óptimo proceso de aprendizaje para el alumnado. La aportación de las investigaciones de Wanzek y Vaughn (2007) indican que el alumnado que recibe una intervención adecuada y temprana avanza de modo más apropiado y consistente en su desarrollo intelectual y, consiguientemente, en el logro de aprendizajes.

La primera fase de la Educación Infantil se inicia con la grafomotricidad, periodo en el que se adquieren las primeras pautas para conseguir un apropiado hábito escritor. La grafomotricidad establece la expresión de una representación del sistema ortográfico que permite seguir un patrón como referencia para alcanzar la forma y la parte de algunas letras (Rius, 2003; Sellés y Martínez, 2008). Para obtener la palabra escrita y su posterior consolidación del hábito escritor, se necesita cambiar la ortografía en sonidos, examinar la estructura del sonido del habla, simbolizada por la escritura (Abbott, Berninger y Fayol, 2010). La importancia de la adquisición de hábitos de escritura la avalan investigaciones, tales como Defior y Serrano (2005), Nemirovsky (1999) y Fons (1999).

\subsection{Hábitos tecnológicos y relacionados con el ejercicio físico}

El análisis factorial realizado para determinar la validez de constructo de la escala aplicada al profesorado encuestado establece relación positiva entre los ítems que valoran la importancia de los hábitos relativos al ejercicio físico y los tecnológicos, encuadrándose en el mismo factor. 
Si importante es el desarrollo del discurso interno del niño y su comunicación con el exterior, no lo es menos la posibilidad de adquirir actitudes y rutinas que le permitan gestionar en un futuro las nuevas tecnologías y la consecución de un desarrollo adecuado de su cuerpo, hablamos pues de los hábitos tecnológicos y aquellos que se dirigen hacia la práctica del deporte. Buceta (2004) y Delgado (2007) aluden a las ventajas del ejercicio físico y establecen las características que debe poseer el deporte escolar para que pueda ser considerado educativo. Los deportes, sobre todo los de equipo, favorecen la participación, la autonomía y el desarrollo de capacidades perceptivo-motoras. Cruz (1997), Gutiérrez (2003) y Monjas (2006) consideran que la escuela debe crear aquellas situaciones educativas y deportivas que fomenten la consolidación de conductas saludables. Sin embargo, Torres (1999) y Gutiérrez (2000) indican que, para que estas actuaciones lleguen a traducirse en hábitos, es necesaria la utilización de buenos programas de actividad física en la escuela, que aporten los conocimientos y las destrezas básicas que desarrollen hábitos hacia la práctica de la actividad física a lo largo de toda la vida del alumnado.

Por consiguiente, cuando hablamos de hábitos tecnológicos nos referimos a la posibilidad de propiciar en el futuro el acceso a las plataformas tecnológicas, donde previamente se han adquirido las pericias y mecanismos necesarios para su adecuada utilización. En las realidades educativas actuales, el aprendizaje se encuentra en proceso de cambio, incluyendo nuevas situaciones menos formales (Solomon y Schrum, 2007; McLoughlin, 2010). Este cambio permite al alumno gestionar de forma diferente los medios tecnológicos, donde descubren e investigan conocimientos curriculares y significativos a través de las redes de comunicación (McLoughlin y Lee, 2007; Valjataga, Pata y Tammets, 2011). Por ello, los docentes de Educación Infantil han de facilitar aquellas estrategias, herramientas y cambios conceptuales que permitan al alumnado interactuar a través de los medios tecnológicos, advirtiéndoles de los inconvenientes y ventajas que ofrecen (Sevillano, 2007). Esto no implica la sustitución de los sistemas aplicados anteriormente, sino proyectar un nuevo enfoque donde la utilización de las TIC (tecnologías de información y comunicación) se inicie en la infancia y trascurra durante todo el ciclo vital (Adell y Castañeda, 2010). Por todo ello, el docente debe contribuir al uso crítico y adecuado de las nuevas tecnologías, tan incipientes en nuestra actual sociedad, considerando necesaria la utilización de las nuevas tecnologías como un recurso útil y práctico para potenciar el proceso de enseñanza y aprendizaje. Además, debe despertar el interés por afianzar hábitos deportivos tales como el gusto por el ejercicio físico, la no competitividad y el disfrute por ayudar a los demás.

\subsection{Hábitos de bigiene y autonomía personal}

Finalmente, los hábitos de cuidado, higiene y autonomía personal responden a aquellos comportamientos y actitudes para alcanzar la consecución de pautas apropiadas por el alumnado, a fin de alcanzar su desenvolvimiento de forma independiente en el contexto que se encuentran inmersos. Así pues, el aprendizaje 

INCIDENCIA DE LOS HÁBITOS EDUCATIVOS EN EL APRENDIZAJE DEL ALUMNADO DE EDUCACIÓN INFANTIL

se puede adquirir a través de las diferentes formas de razonar de modo eficaz, por parte del alumnado, desde las edades tempranas (Entwistle, 2000). Por ello, el mejor momento para iniciar la adquisición de la autonomía personal es el periodo infantil. Estos acontecimientos se encuentran ligados a factores que favorecerán la madurez personal futura. Para ello se han de planificar actividades y metodologías que regulen el sueño y descanso personal, que organicen aquellos aspectos de higiene personal, que sean capaces de gestionar las tareas de forma autónoma, imprescindibles todas ellas para su formación. No obstante, el profesorado debe manifestar preocupación por los hábitos afianzados no deseados (mala alimentación, insuficiente higiene corporal, distorsión de hábitos de sueño-vigilia) como reto para modificarlos. Simultáneamente, debe organizar actividades para que el alumnado ponga en práctica acciones de colaboración, cooperación y ayuda entre iguales, logrando la adquisición de los hábitos previamente descritos.

Concluidas las reflexiones anteriores, se plantea el siguiente problema de investigación: ¿Qué opinión tiene el profesorado de Educación Infantil sobre la incidencia de los hábitos educativos en la adquisición de aprendizajes por su alumnado?

\section{Metodología y diseño de la inVeStigación}

El diseño metodológico seguido en esta investigación se basa en un modelo descriptivo-exploratorio, que permite conocer cuál es el comportamiento de la opinión del profesorado consultado sobre la incidencia de los hábitos educativos del currículum de Educación Infantil en el proceso de aprendizaje del alumnado de tres a seis años. Los estadígrafos de tendencia central utilizados son: media, mediana y moda, los de dispersión: desviación típica, y razón de equivalencia.

Investigaciones recientes llevadas a cabo en países con altos niveles de desarrollo educativo muestran la importancia de las opiniones de los docentes para la obtención de buenos resultados en los aprendizajes del alumnado, así lo ponen de manifiesto Barber y Mourshed (2008), entre otros autores.

\subsection{Objetivos de la investigación}

Objetivo general

Conocer la opinión que tiene el profesorado consultado sobre la influencia de los hábitos educativos englobados en el Currículum de Educación Infantil, en la adquisición de aprendizajes.

A partir del objetivo general formulado, pretendemos dar respuesta a los siguientes objetivos específicos:

- Determinar los distintos tipos de hábitos con los que trabaja el profesorado en el segundo ciclo de Educación Infantil. 
MARÍA MARTÍNEZ REDONDO, MIGUEL PÉREZ FERRA Y BEATRIZ SIERRA Y ARIZMENDIARRIETA INCIDENCIA DE LOS HÁBITOS EDUCATIVOS EN EL APRENDIZAJE DEL ALUMNADO DE EDUCACIÓN INFANTIL

- Conocer la opinión del profesorado sobre los hábitos que tienen mayor incidencia en la adquisición de aprendizaje por el alumnado.

- Valorar la opinión del profesorado encuestado sobre la incidencia de los hábitos morales, conductuales y sociales en el aprendizaje de su alumnado.

- Determinar la opinión del profesorado encuestado sobre la incidencia de los hábitos ambientales, alimentación y de vida saludable en el aprendizaje de su alumnado.

- Establecer la opinión del profesorado encuestado sobre la incidencia de los hábitos de lectura y escrita en el aprendizaje de su alumnado.

- Valorar la opinión del profesorado encuestado sobre la incidencia de los hábitos tecnológicos y deportivos en la adquisición de aprendizajes del alumnado.

- Conocer la opinión del profesorado encuestado sobre la incidencia de los hábitos de autonomía y cuidado personal en la adquisición de aprendizajes de su alumnado.

\subsection{Hipótesis exploratorias}

1) No hay diferencias estadísticamente significativas en las opiniones del profesorado de Educación Infantil, atendiendo al sexo de los docentes, respecto a que los hábitos educativos inciden en adquisición de aprendizajes.

2) No hay diferencias estadísticamente significativas en las opiniones del profesorado de Educación Infantil, atendiendo a los años de experiencia docente de los docentes, respecto a que los hábitos educativos inciden en la adquisición de aprendizajes.

3) No hay diferencias estadísticamente significativas en las opiniones del profesorado de Educación Infantil, atendiendo a la titulación de más alto rango que poseen los docentes, respecto a que los hábitos educativos inciden en la adquisición de aprendizajes.

4) No hay diferencias estadísticamente significativas en las opiniones del profesorado de Educación Infantil, atendiendo al tiempo de permanencia del profesorado en su centro actual, respecto a que los hábitos educativos inciden en adquisición de aprendizajes.

5) No hay diferencias estadísticamente significativas en las opiniones del profesorado de Educación Infantil, atendiendo a la naturaleza del tipo de centro docente en el que trabaja, respecto a que los hábitos educativos inciden en la adquisición de aprendizajes.

6) No hay diferencias estadísticamente significativas en las opiniones del profesorado de Educación Infantil, atendiendo a la localización geográfica del centro educativo en el que trabajan, respecto a que los hábitos educativos inciden en la adquisición de aprendizajes. 


\subsection{Población y muestra}

La población la integran cien profesores y profesoras de Educación Infantil de las demarcaciones geográficas de: Úbeda, Sierra de Segura, Las Villas y El Condado. Se ha considerado más operativo realizar la investigación con la población, por el número de participantes, suministrando información a todos los integrantes de la población; es decir, cien participantes.

\subsection{Elaboración y aplicación del instrumento}

Con posterioridad a la revisión de la bibliografía específica sobre el tema, así como diferentes instrumentos relacionados con la incidencia de los hábitos en el desarrollo de aprendizaje, y siguiendo los objetivos explicitados en la investigación, punto de referencia para confeccionar el cuestionario y aspectos que constituyeron su eje vertebrador, el instrumento de recogida de información elaborado fue una escala aditiva tipo Likert con cuatro opciones de respuestas para evitar el efecto "centralidad" en las respuestas, utilizando los pasos que se indican en la Figura 2.

FIGURA 2

Fases en la elaboración del instrumento de recogida de información

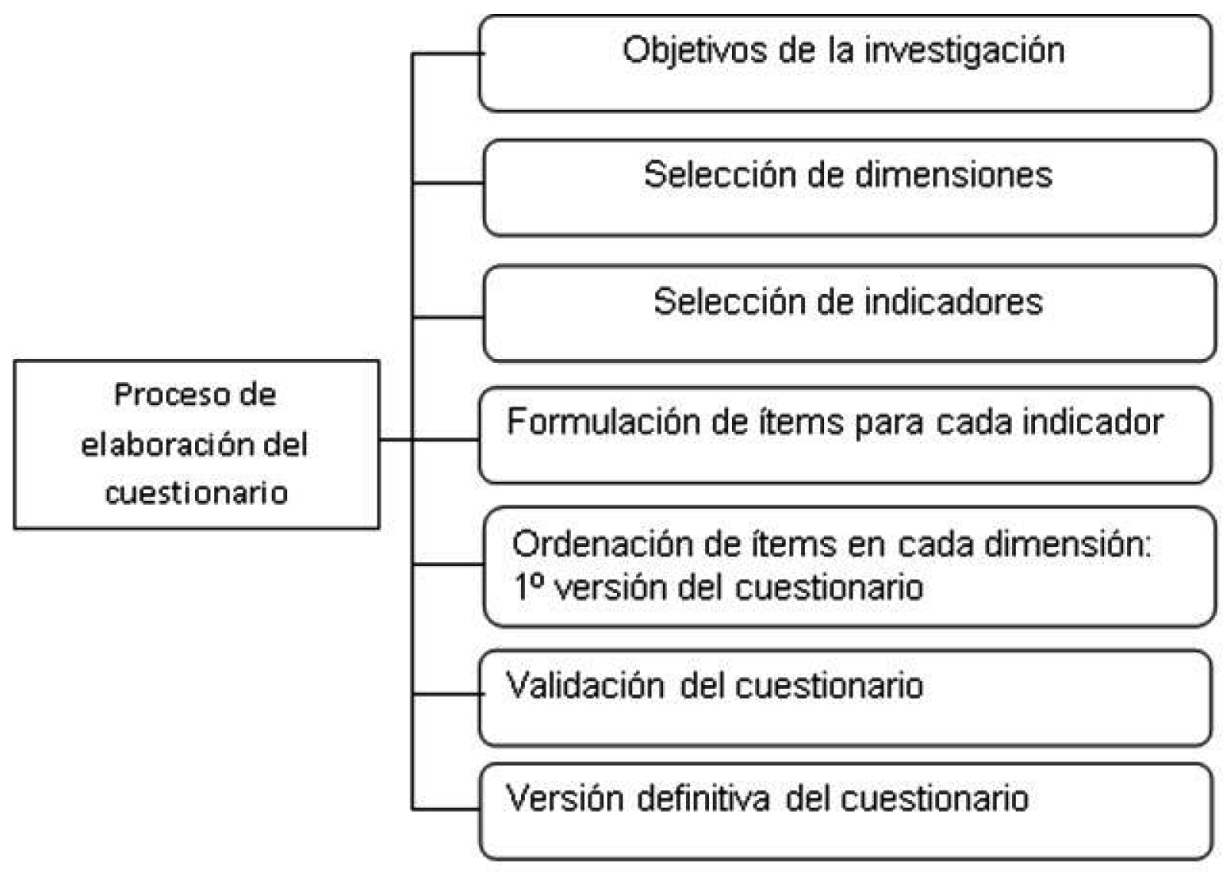


MARÍA MARTÍNEZ REDONDO, MIGUEL PÉREZ FERRA Y BEATRIZ SIERRA Y ARIZMENDIARRIETA INCIDENCIA DE LOS HÁBITOS EDUCATIVOS EN EL APRENDIZAJE DEL ALUMNADO DE EDUCACIÓN INFANTIL

\subsection{Estudio de fiabilidad y validez}

Para conocer si el rasgo de la escala es representativo del universo temático que pretende valorar, determinamos la validez de contenido. La escala se sometió a la validación de 20 expertos, valorando de 1 a 5 cada ítem, considerando la pertinencia del ítem con el rasgo a valorar, claridad del lenguaje y suficiencia del número de cuestiones formuladas, quedando reducidos los ítems de 60 a 40 tras esta primera valoración.

La varianza total explicada supone el 48,157\%, más que suficiente para definir el rasgo de la escala.

TABLA 1

Porcentaje de varianza total explicada para los cinco factores de la escala Likert

\begin{tabular}{|c|c|c|c|c|c|c|}
\hline \multirow{2}{*}{ Componentes } & \multicolumn{3}{|c|}{ Autovalores iniciales } & \multicolumn{3}{c|}{ Suma de las saturaciones al cuadrado de la rotación } \\
\cline { 2 - 7 } & Total & $\begin{array}{l}\text { \% de la } \\
\text { varianza }\end{array}$ & \% acumulado & Total & \% de la varianza & \% acumulado \\
\hline 1 & 8,499 & 21,248 & 21,248 & 4,453 & 11,133 & 11,133 \\
\hline 2 & 2,992 & 7,481 & 28,729 & 4,110 & 10,274 & 21,407 \\
\hline 3 & 2,675 & 6,688 & 35,417 & 3,419 & 8,549 & 29,955 \\
\hline 4 & 2,070 & 5,175 & 40,592 & 3,118 & 7,794 & 37,749 \\
\hline 5 & 1,810 & 4,526 & 45,118 & 2,947 & 7,368 & 45,118 \\
\hline
\end{tabular}

La medida de adecuación muestral aportó un coeficiente de correlación de 0,7000 y la prueba de esfericidad de Bartlett aporto una sig. $=0,000$, razón por la que se rechaza la hipótesis nula, ambos indicadores permitieron realizar la validez de constructo, que se obtuvo mediante rotación varimax, con un índice de discriminación de $\mathrm{K} \geq 0,05$, quedando la escala integrada por 40 ítems, distribuidos en 5 factores, atendiendo a los resultados aportados por el gráfico de sedimentación y reflejados en la Tabla 3: componente n. $\stackrel{0}{1}$, hábitos morales, conductuales y sociales; componente n.. 2, hábitos ambientales, alimenticios; componente n. 3 , hábitos lectoescritores; componente n.ำ 4, hábitos tecnológicos y deportivos; componente n. ${ }^{\circ} 5$, hábitos de higiene y autonomía personal.

La fiabilidad del instrumento se determinó mediante el Alpha de Cronbach para la totalidad, aportando un Alpha $=0,915$, por lo que la consistencia interna, es decir, el grado en que las respuestas son independientes de las circunstancias accidentales de la investigación es muy elevado.

\subsection{Variables de la investigación}

- Variables descriptivas:

- De carácter demográfico: sexo del profesorado, edad, experiencia docente, titulación de más alto grado que posee el docente.

- De centro: tiempo de permanencia en el mismo centro, tipo de centro educativo y localización del centro. 

INCIDENCIA DE LOS HÁBITOS EDUCATIVOS EN EL APRENDIZAJE DEL ALUMNADO DE EDUCACIÓN INFANTIL

- Variable independiente: Incidencia de los hábitos educativos en el aprendizaje del alumnado de Educación Infantil.

\section{ANÁlisis DE LOS RESUlTADOS DE LA INVESTIGACIÓN}

\subsection{Estudio descriptivo}

En relación a los hábitos morales, conductuales y sociales, el profesorado encuestado pone de manifiesto la incidencia de estos hábitos en el aprendizaje del alumnado, concretamente el $26 \%$ de los encuestados manifiesta estar "de acuerdo", mientras que el 73\% se posiciona en "muy de acuerdo", lo que supone que el 99\% del profesorado consultado considera que esos hábitos son importantes para facilitar el estudio. Así queda ratificado por la media de las opiniones del profesorado consultado que se cifra en 3,71, "muy de acuerdo"; por la homogeneidad de la distribución de las opiniones, ya que la diferencia entre el punto central de esas opiniones (mediana) y la media es casi imperceptible (media 3,71, mediana, 3,75), circunstancia que pone de manifiesto la escasa incidencia en la media de valores extremos, a los que es muy sensible. Esta evidencia, junto al hecho de que la desviación típica es $\alpha=0,32014$, confirman que nos hallamos ante una opinión bastante homogénea, cuyas opciones de respuesta se concentran entre "muy de acuerdo" y "acuerdo". La opción de respuesta más elegida, moda, es 4, "muy de acuerdo".

TABLA 2

Frecuencias, porcentajes, medidas de tendencia central y dispersión de las opiniones del profesorado de Educación Infantil respecto a los hábitos morales, conductuales y sociales

\begin{tabular}{|c|c|c|c|c|c|c|c|}
\hline \multicolumn{3}{|c|}{ Opciones de respuesta } & Frecuencias & \multicolumn{2}{|c|}{ Porcentajes } & $\begin{array}{c}\text { Porcentajes } \\
\text { válidos }\end{array}$ & $\begin{array}{l}\text { Porcentajes } \\
\text { acumulado } \\
\text { s }\end{array}$ \\
\hline \multicolumn{3}{|c|}{ Completamente en desacuerdo (1) } & 0 & \multicolumn{2}{|c|}{0} & 0 & 0 \\
\hline \multicolumn{3}{|c|}{ En desacuerdo (2) } & 1 & \multicolumn{2}{|c|}{1} & 1 & 1 \\
\hline \multicolumn{3}{|c|}{ De acuerdo (3) } & 26 & \multicolumn{2}{|c|}{26} & 26 & 27 \\
\hline \multicolumn{3}{|c|}{ Completamente de acuerdo (4) } & 73 & \multicolumn{2}{|c|}{73} & 73 & 100 \\
\hline N. Válidos & N. Perdidos & Media & Mediana & Moda & $\begin{array}{l}\text { De } \\
\text { tipi }\end{array}$ & Máximo & Mínimo \\
\hline 100 & 0 & 3,7113 & 3,7500 & 4,00 &, 320 & 4 & 3 \\
\hline
\end{tabular}

Las respuestas facilitadas por el profesorado con relación a los hábitos ambientales, alimenticios y saludables que conforman la dimensión n. $\stackrel{2}{2}$ revelan que el profesorado muestra un acuerdo casi total con relación a la incidencia de los mencionados hábitos respecto al aprendizaje del alumnado, suma "de acuerdo" $40 \%$ y "muy de acuerdo" 58\%; es decir, el 98\% del profesorado encuestado considera los hábitos ambientales y alimenticios muy significativos para el desarrollo de aprendizajes en los alumnos de 3 a 6 años. Las medidas de tendencia central evidencian que estamos ante opiniones bastante uniformes, dado que la media es 
3,56, la mediana 3,62 y la moda, puntuación más elegida, 4. Las medidas de dispersión ratifican lo manifestado, ya que la desviación típica se sitúa en $\alpha=0,37$, lo que supone que el $68 \%$ de la opinión del profesorado se halla localizada entre el "de acuerdo" y el "muy de acuerdo", siendo la puntuación más elegida "muy de acuerdo" 4; y el máximo y mínimo, 4 y 3, respectivamente.

\section{GRÁFICO 1}

Distribución de porcentajes de las opiniones del profesorado encuestado respecto a la incidencia de los hábitos morales, conductuales y sociales en el aprendizaje de niños/as de 3 a 6 años

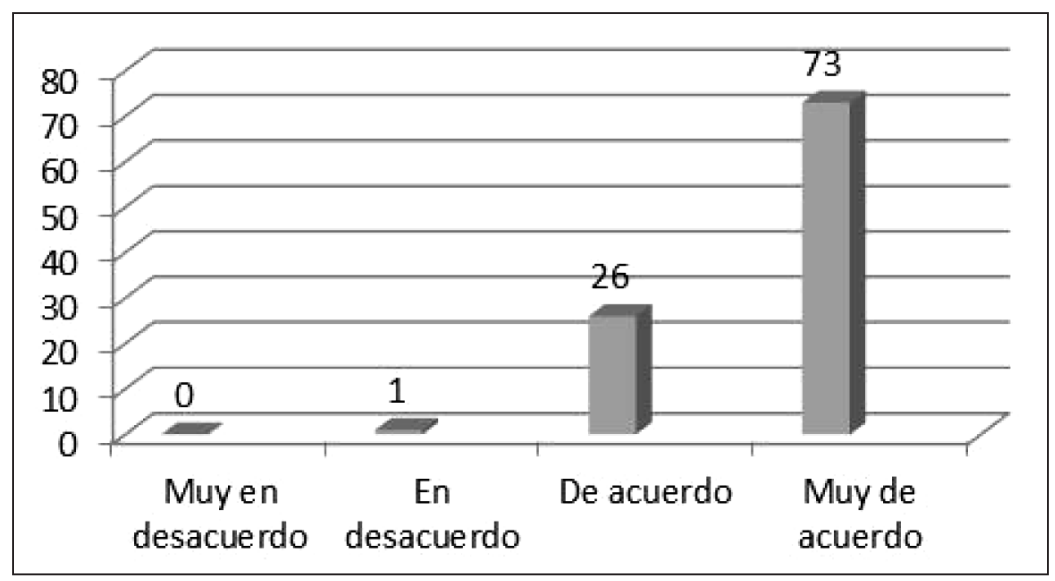

TABLA 3

Frecuencias, porcentajes, medidas de tendencia central y dispersión de las opiniones del profesorado de Educación Infantil respecto a los hábitos ambientales y alimentarios

\begin{tabular}{|c|c|c|c|c|c|c|c|}
\hline \multicolumn{3}{|c|}{ Opciones de respuesta } & Frecuencias & \multicolumn{2}{|c|}{ Porcentajes } & $\begin{array}{l}\text { Porcentajes } \\
\text { válidos }\end{array}$ & $\begin{array}{l}\text { Porcentajes } \\
\text { acumulados }\end{array}$ \\
\hline \multicolumn{3}{|c|}{ Completamente en desacuerdo (1) } & 0 & \multicolumn{2}{|l|}{0} & 0 & 0 \\
\hline \multicolumn{3}{|c|}{ En desacuerdo (2) } & 2 & \multicolumn{2}{|l|}{2} & 2 & 2 \\
\hline \multicolumn{3}{|c|}{ De acuerdo (3) } & 40 & \multicolumn{2}{|l|}{40} & 40 & 42 \\
\hline \multicolumn{3}{|c|}{ Completamente de acuerdo (4) } & 58 & 58 & & 58 & 100 \\
\hline N. Válidos & N. Perdidos & Media & Mediana & Moda & $\begin{array}{l}\text { Desv. } \\
\text { tipica }\end{array}$ & Máximo & Minimo \\
\hline 100 & 0 & 3,5638 & 3,6250 & 4,00 &, 37416 & 4 & 3 \\
\hline
\end{tabular}


MARÍA MARTÍNEZ REDONDO, MIGUEL PÉREZ FERRA Y BEATRIZ SIERRA Y ARIZMENDIARRIETA INCIDENCIA DE LOS HÁBITOS EDUCATIVOS EN EL APRENDIZAJE DEL ALUMNADO DE EDUCACIÓN INFANTIL

GRÁFICO 2

Distribución de porcentajes de las opiniones del profesorado encuestado respecto a la incidencia de los hábitos ambientales y alimentarios en el aprendizaje de niños/as de 3 a 6 años

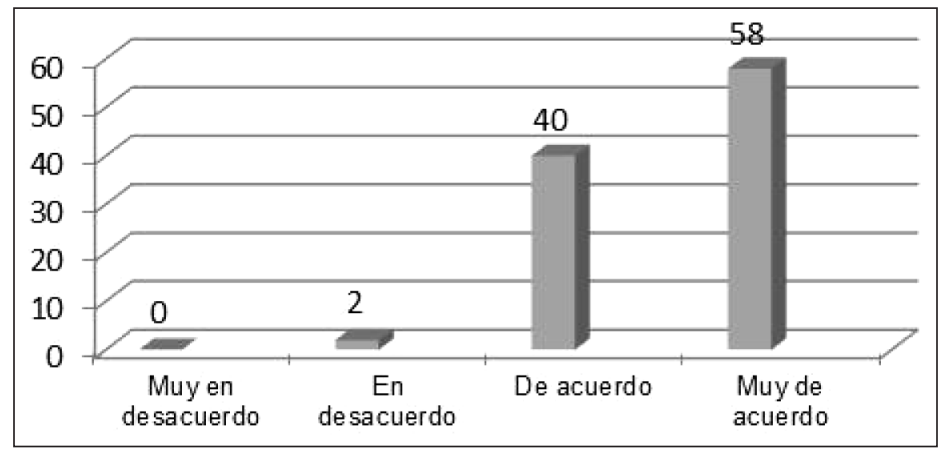

Considerando la totalidad de los ítems representativos, los hábitos de lectura y escritura que comprenden la dimensión n. 3 , se advierte que el profesorado muestra un acuerdo significativo con relación a la incidencia de los hábitos lectoescritores en el aprendizaje del alumnado, suma "de acuerdo" 30\% y "completamente de acuerdo" 68\%. Tan solo el 2\% de los encuestados se posiciona en "desacuerdo". Las medidas de tendencia central muestran que estamos ante opiniones bastantes similares, evidenciadas por la casi inapreciable diferencia existente entre la media $(3,62)$ y la mediana $(3,66)$. El valor de la desviación típica $(0,373)$ pone de manifiesto la escasa dispersión de las opiniones manifestadas por los docentes, aunque algo mayor que en otras dimensiones. De todos modos, el 68\% del profesorado encuestado se posiciona entre "muy de acuerdo" y "de acuerdo". En esta ocasión el "muy de acuerdo" duplica ampliamente al "de acuerdo", y la suma de ambas opciones de respuesta se vuelve a situar por encima del 90\%, concretamente en el 98\% de respuestas, siendo el máximo y el mínimo, respectivamente, 4 y 3, y la opción de respuesta más elegida (moda), "muy de acuerdo", 4.

TABLA 4

Frecuencias, porcentajes, medidas de tendencia central y dispersión de las opiniones del profesorado de Educación Infantil respecto a los hábitos lectoescritores

\begin{tabular}{|c|c|c|c|c|c|c|c|c|}
\hline \multicolumn{3}{|c|}{ Opciones de respuesta } & Frecuencias & \multicolumn{2}{|c|}{ Porcentajes } & & $\begin{array}{l}\text { rcentajes } \\
\text { álidos }\end{array}$ & $\begin{array}{l}\text { Porcentajes } \\
\text { acumulados }\end{array}$ \\
\hline \multicolumn{3}{|c|}{ Completamente en desacuerdo (1) } & 0 & \multicolumn{2}{|l|}{0} & & 0 & 0 \\
\hline \multicolumn{3}{|c|}{ En desacuerdo (2) } & 2 & \multicolumn{2}{|l|}{2} & & 2 & 2 \\
\hline \multicolumn{3}{|c|}{ De acuerdo (3) } & 30 & \multicolumn{2}{|l|}{30} & & 30 & 32 \\
\hline \multicolumn{3}{|c|}{ Completamente de acuerdo (4) } & 68 & 68 & & & 68 & 100 \\
\hline N. Válidos & N. Perdidos & Media & Mediana & Moda & & & Máximo & Mínimo \\
\hline 100 & 0 & 3,6267 & 3,6667 & 4,00 & & 314 & 4 & 3 \\
\hline
\end{tabular}


MARÍA MARTÍNEZ REDONDO, MIGUEL PÉREZ FERRA Y BEATRIZ SIERRA Y ARIZMENDIARRIETA INCIDENCIA DE LOS HÁBITOS EDUCATIVOS EN EL APRENDIZAJE DEL ALUMNADO DE EDUCACIÓN INFANTIL

GRÁFICO 3

Distribución de porcentajes de las opiniones del profesorado encuestado respecto a la incidencia de los hábitos lectoescritores en el aprendizaje de niños/as de 3 a 6 años

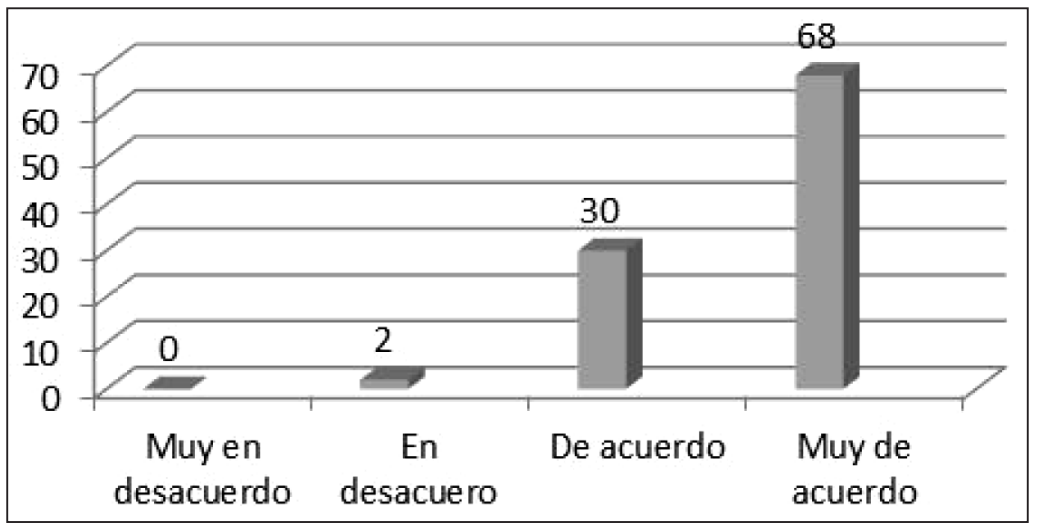

Es tradición relacionar los hábitos tecnológicos y deportivos, así lo ponen de manifiesto autores como Gapllonch (2005) o Generelo (2010) entre otros, cuando afirman que las TIC acentúan el protagonismo del niño o niña en el desarrollo de su actividad física. Respecto a esta dimensión, el profesorado se posiciona de modo casi unánime, respecto a que los hábitos favorecen la adquisición de aprendizajes variados. Los docentes responden con un acuerdo casi generalizado, el 96\% de los encuestados comparten esta opinión, distribuida entre el 58\% "de acuerdo" y el 38\% "muy de acuerdo". La media de las respuestas dadas se sitúa en el 3,47, lo que supone que se encuentra en "de acuerdo", en el mismo nivel de respuesta se encuentra el punto central, la mediana, 3 .

Hay mayor diferencia entre la media $(3,4275)$ respecto al punto central de las posibles opciones de respuesta, mediana $(3,00)$, lo cual pone de manifiesto que la media está más afectada que en otras dimensiones por puntuaciones más extremas, aspecto evidenciado por la desviación típica $(\alpha=0,43125)$. Esta circunstancia plantea un mayor nivel de dispersión en las opiniones del profesorado, aunque no es menos cierto que el 68\% de las opiniones emitidas por los docentes se sitúan en torno a "de acuerdo"; es decir, en torno a la puntuación 3, siendo la puntuación más elegida. Aunque el número de respuestas en torno a "en desacuerdo" sigue siendo irrelevante, cuatro profesores, no es menos cierto que en esta dimensión alcanza mayor número que en las restantes.

De todos modos, y considerando una dispersión algo mayor en las respuestas de los docentes encuestados, se sigue observando una línea de regularidad en torno a "de acuerdo", en consonancia con la dimensión de hábitos tecnológicos y deportivos. 
164 MARÍA MARTÍNEZ REDONDO, MIGUEL PÉREZ FERRA Y BEATRIZ SIERRA Y ARIZMENDIARRIETA INCIDENCIA DE LOS HÁBITOS EDUCATIVOS EN EL APRENDIZAJE DEL ALUMNADO

DE EDUCACIÓN INFANTIL

TABLA 5

Frecuencias, porcentajes, medidas de tendencia central y dispersión de las opiniones del profesorado de Educación Infantil respecto a los hábitos tecnológicos y deportivos

\begin{tabular}{|c|c|c|c|c|c|c|c|}
\hline \multicolumn{3}{|c|}{ Opciones de respuesta } & Frecuencias & Porcentajes & \multicolumn{2}{|c|}{$\begin{array}{c}\text { Porcentajes } \\
\text { válidos }\end{array}$} & $\begin{array}{l}\text { Porcentajes } \\
\text { acumulados }\end{array}$ \\
\hline \multicolumn{2}{|c|}{ Completamente en desacuerdo (1) } & & 0 & 0 & & 0 & 0 \\
\hline \multicolumn{2}{|c|}{ En desacuerdo (2) } & & 4 & 4 & & 4 & 4 \\
\hline \multicolumn{2}{|c|}{ De acuerdo (3) } & & 58 & 58 & & 58 & 62 \\
\hline \multicolumn{2}{|c|}{ Completamente de acuerdo (4) } & & 38 & 38 & & 38 & 100 \\
\hline N. Válidos & N. Perdidos & Media & Median & Moda & $\begin{array}{l}\text { Desv. } \\
\text { típica }\end{array}$ & Máximo & Mínimo \\
\hline 100 & 0 & 3,4275 & 3,5000 & 3,00 & 43125 & 4 & 3 \\
\hline
\end{tabular}

GRÁFICO 4

Distribución de porcentajes de las opiniones del profesorado encuestado, respecto a la incidencia de los hábitos tecnológicos y deportivos en el aprendizaje de niños/as de 3 a 6 años

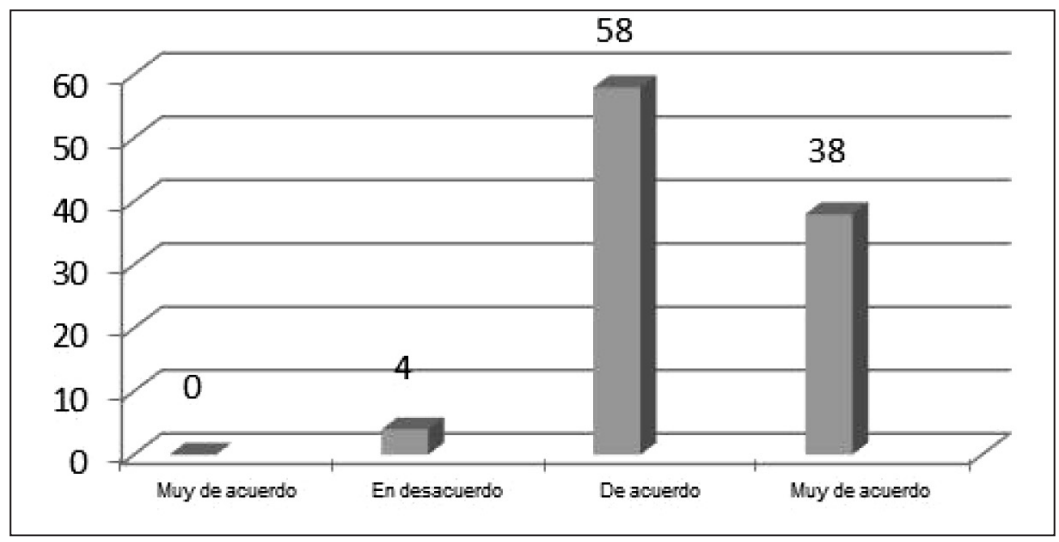

La quinta dimensión analizada responde a los hábitos de cuidado, higiene y autonomía personal. El profesorado consultado sigue opinando mayoritariamente en torno al acuerdo, 52\% "de acuerdo", 47\% "muy de acuerdo" y tan solo hay un profesor que se manifiesta "en desacuerdo". En esta ocasión la dispersión de las opiniones de los docentes es menor que en los hábitos tecnológicos y deportivos, media $(3,5775)$ y mediana $(3,5000)$. Del mismo modo, el hecho de que la desviación típica sea $\alpha=0,33276$ plantea una menor dispersión de las opiniones del profesorado respecto a otras dimensiones de la escala. Dicho lo cual, el 99\% del profesorado encuestado manifiesta estar de acuerdo respecto a que los hábitos de cuidado, higiene y autonomía personal, sobre todo este último, atendiendo a 
MARÍA MARTÍNEZ REDONDO, MIGUEL PÉREZ FERRA Y BEATRIZ SIERRA Y ARIZMENDIARRIETA INCIDENCIA DE LOS HÁBITOS EDUCATIVOS EN EL APRENDIZAJE DEL ALUMNADO DE EDUCACIÓN INFANTIL

las respuestas dadas en la escala, inciden positivamente en el aprendizaje de los alumnos del segundo ciclo de Educación Infantil.

\section{GRÁFICO 5}

Distribución de porcentajes de las opiniones del profesorado encuestado, respecto a la incidencia de los hábitos de cuidado, higiene y autonomía personal en relación a su incidencia en el aprendizaje de niños/as de 3 a 6 años

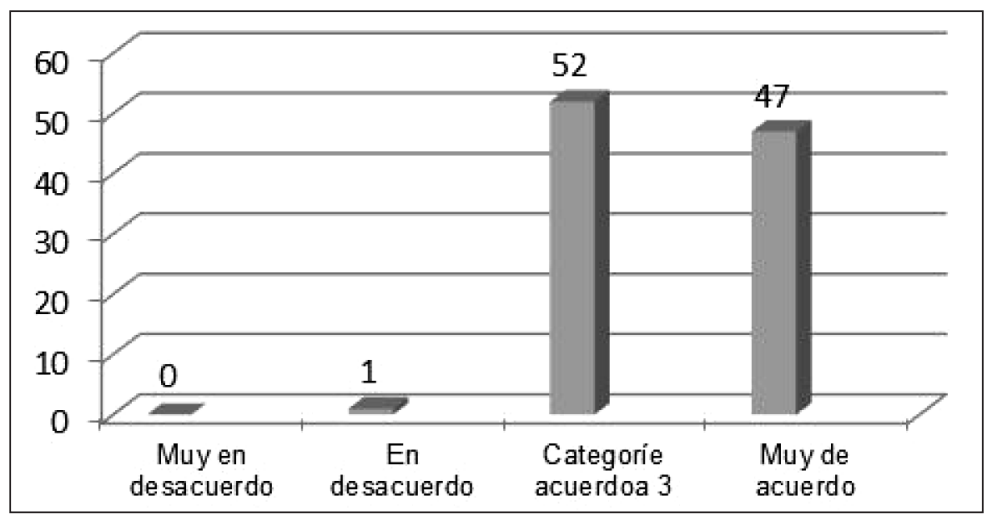

TABLA 6

Frecuencias, porcentajes, medidas de tendencia central y dispersión de las opiniones del profesorado de Educación Infantil respecto a los hábitos de cuidado, higiene y autonomía personal

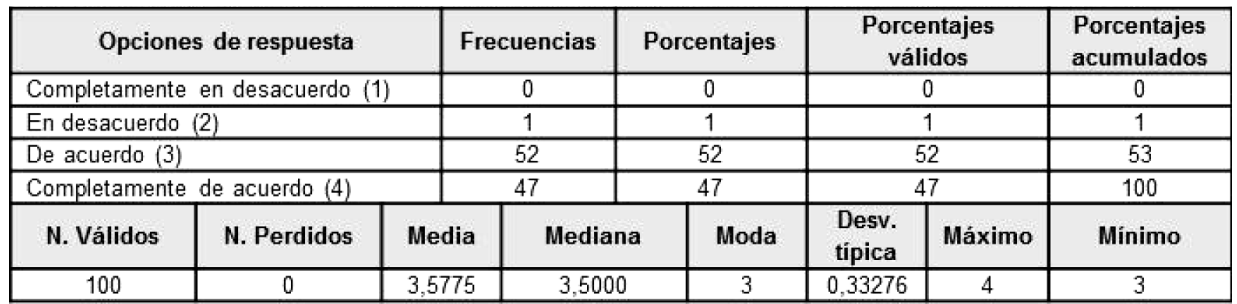

En último lugar hacemos la estimación de la valoración del profesorado en relación a la totalidad de los factores que integran la variable dependientes; es decir, las opiniones del profesorado respecto a si los hábitos educativos considerados en su totalidad inciden, o no, positivamente en la adquisición de aprendizajes por los niños y niñas de 3 a 6 . El análisis de los resultados reflejados en la Tabla 7 y el Gráfico 6 plantea que el 100\% de encuestados manifiestan acuerdo respecto a que los hábitos educativos considerados en la investigación inciden positivamente en la adquisición de aprendizajes, opiniones distribuidas del siguiente modo: el 40\% 
manifiestan estar "de "acuerdo" y el 60\% "muy de acuerdo". No habiendo ninguna respuesta en torno al desacuerdo.

Los datos aportados por las medidas de dispersión evidencian que la desviación típica es $\alpha=0,239$; es decir, que los profesores han manifestado opiniones entre "de acuerdo" y "muy de acuerdo", máximo y mínimo (4) y (3). Determinada la razón de intervalo $(\alpha \pm \mathrm{x})$, los resultados alcanzados también se posicionan entre "muy de acuerdo" $(4,000)$ y "de acuerdo" $(3,1275)$, 95\% de la curva. También la opción de respuesta más elegida es "muy de acuerdo" (moda $=4)$; por consiguiente estamos antes opiniones muy homogéneas que se sitúan entre "de acuerdo" y "muy de acuerdo".

\section{GRÁFICO 6}

Distribución de porcentajes de las opiniones del profesorado encuestado, respecto a la incidencia de los hábitos educativos en relación a su incidencia en los aprendizajes de niños y niñas de 3 a 6 años

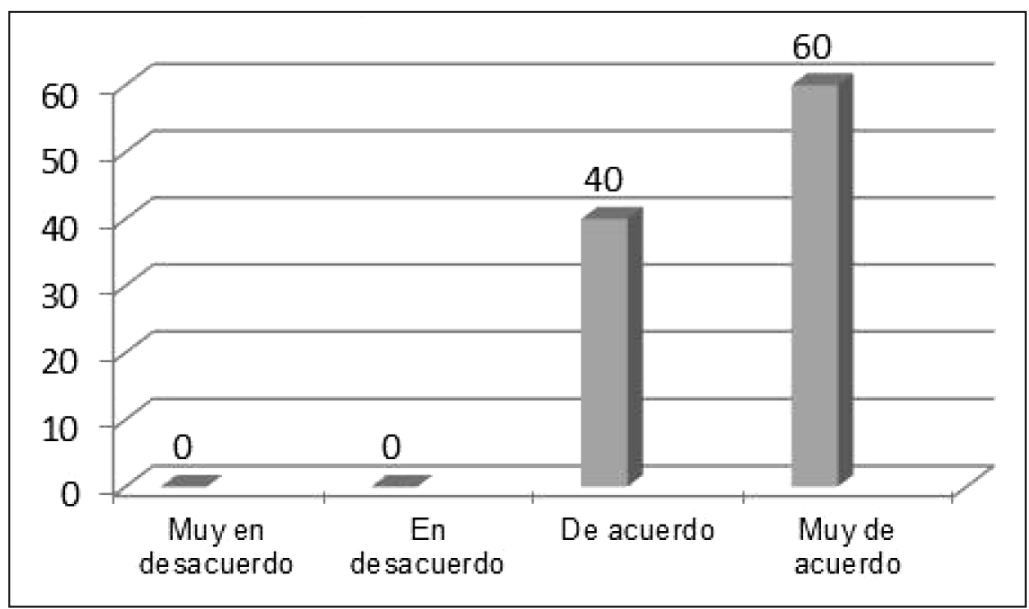

TABLA 7

Frecuencias, porcentajes, medidas de tendencia central y dispersión de las opiniones del profesorado de Educación Infantil respecto a la totalidad de los hábitos educativos

\begin{tabular}{|c|c|c|c|c|c|c|c|}
\hline \multicolumn{3}{|c|}{ Opciones de respuesta } & Frecuencias & Porcentajes & Po & $\begin{array}{l}\text { centajes } \\
\text { álidos }\end{array}$ & $\begin{array}{l}\text { Porcentajes } \\
\text { acumulados }\end{array}$ \\
\hline \multicolumn{3}{|c|}{ Completamente en desacuerdo (1) } & 0 & 0 & & 0 & 0 \\
\hline \multicolumn{3}{|c|}{ En desacuerdo (2) } & 0 & 0 & & 0 & 0 \\
\hline \multicolumn{3}{|c|}{ De acuerdo (3) } & 40 & 40 & & 40 & 40 \\
\hline \multicolumn{3}{|c|}{ Completamente de acuerdo (4) } & 60 & 60 & & 60 & 100 \\
\hline N. Válidos & N. Perdidos & Media & Mediana & Moda & $\begin{array}{l}\text { Desv. } \\
\text { tipica }\end{array}$ & Máximo & Mínimo \\
\hline 100 & 0 & 3,6063 & 3,6250 & 4 & 0,23943 & 4 & 3 \\
\hline
\end{tabular}


MARÍA MARTÍNEZ REDONDO, MIGUEL PÉREZ FERRA Y BEATRIZ SIERRA Y ARIZMENDIARRIETA INCIDENCIA DE LOS HÁBITOS EDUCATIVOS EN EL APRENDIZAJE DEL ALUMNADO DE EDUCACIÓN INFANTIL

\subsection{Estudio exploratorio}

El estudio de referencia incluye la "t" de Student para muestras relacionadas (variable dicotómica sexo) y un análisis de varianza de cada una de las variables descriptivas respecto a la variable dependiente, con un nivel de significación de 0,05 .

"t" de Student

Los datos obtenidos, Tabla 8, plantean que la diferencia entre las medias es de $(-1,70625)$ y el límite aceptable está comprendido entre los valores $(-1,78374)$ y $(-1,62875)$, lo que supone que se acepta que las medidas son diferentes. Siendo el estadístico "t" $=-43,688$ y su significación $p=0,000<0,025$ (contraste bilateral), se rechaza la hipótesis nula de igualdad de medias; por consiguiente, hay diferencias estadísticamente significativas en la opinión del profesorado sobre la incidencia de los hábitos educativos en el estudio de los niños de 3 a 6 años, atendiendo al sexo.

TABLA 8

Prueba de muestras relacionadas (t de Student)

\begin{tabular}{|c|c|c|c|c|c|c|c|c|}
\hline & \multicolumn{5}{|c|}{ Diferencias relacionadas } & \multirow[b]{2}{*}{ t } & \multirow[b]{2}{*}{ gl } & \multirow[b]{2}{*}{$\begin{array}{c}\text { Sig. } \\
\text { (bilateral) }\end{array}$} \\
\hline & Media & $\begin{array}{c}\text { Desv. } \\
\text { Tip. }\end{array}$ & $\begin{array}{c}\text { Error tip. } \\
\text { de la } \\
\text { media }\end{array}$ & $\begin{array}{r}95 \% \text { In } \\
\text { confiar }\end{array}$ & $\begin{array}{l}\text { valo de } \\
\text { para la } \\
\text { acia }\end{array}$ & & & \\
\hline & \multirow[b]{2}{*}{-1.70625} & \multirow[b]{2}{*}{0,39055} & \multirow[b]{2}{*}{0,03906} & Inferior & Superior & \multirow[b]{2}{*}{$-43,688$} & \multirow[b]{2}{*}{99} & \multirow[b]{2}{*}{0,000} \\
\hline $\begin{array}{l}\text { Sexo- } \\
\text { Total }\end{array}$ & & & & $-1,78374$ & $-1,62876$ & & & \\
\hline
\end{tabular}

Los análisis de varianza efectuados para las variables descriptivas: edad (sig. = 0,343), experiencia docente ( $\mathrm{sig} .=0,170)$, titulación de más elevado nivel del profesor $(\operatorname{sig}=0,543)$, tiempo de permanencia en el centro de destino ( $\operatorname{sig} .=0,226$ ), tipo de centro docente en el que trabaja ( halla el centro (sig. $=0,425)$, ponen de manifiesto, Tabla 9, que no hay diferencias estadísticamente significativas entre cada una de las variables enumeradas y la variable dependiente: «incidencia de los hábitos educativos en el aprendizaje de escolares del segundo ciclo de Educación Primaria"; por consiguiente, se cumple en cada una de ellas la hipótesis nula, ya que en todos los casos $0,05<p$. Los resultados ponen de manifiesto que se cumple la hipótesis nula formulada en todas las hipótesis. 
MARÍA MARTÍNEZ REDONDO, MIGUEL PÉREZ FERRA Y BEATRIZ SIERRA Y ARIZMENDIARRIETA INCIDENCIA DE LOS HÁBITOS EDUCATIVOS EN EL APRENDIZAJE DEL ALUMNADO DE EDUCACIÓN INFANTIL

TABLA 9

Anova de un factor para las variables descriptivas de la investigación respecto a la variable dependiente

\begin{tabular}{|c|c|c|c|c|c|c|}
\hline & & $\begin{array}{l}\text { Suma de } \\
\text { cuadrados }\end{array}$ & gl & $\begin{array}{c}\text { Media } \\
\text { cuadrática }\end{array}$ & F & Sig. \\
\hline Edad & $\begin{array}{l}\text { Inter-grupos } \\
\text { Intra-grupos } \\
\text { Total }\end{array}$ & $\begin{array}{r}56.161 \\
100.429 \\
156.590\end{array}$ & $\begin{array}{l}33 \\
66 \\
99\end{array}$ & $\begin{array}{l}1,702 \\
1,522\end{array}$ & 1,118 & 0,343 \\
\hline Experiencia docente & $\begin{array}{l}\text { Inter-grupos } \\
\text { Intra-grupos } \\
\text { Total }\end{array}$ & $\begin{array}{r}58,037 \\
88,153 \\
146,190 \\
\end{array}$ & $\begin{array}{l}33 \\
66 \\
99\end{array}$ & $\begin{array}{l}1,759 \\
1,336\end{array}$ & 1,317 & 0,170 \\
\hline $\begin{array}{l}\text { Titulación de más alto } \\
\text { grado }\end{array}$ & $\begin{array}{l}\text { Inter-grupos } \\
\text { Intra-grupos } \\
\text { Total } \\
\end{array}$ & $\begin{array}{l}18,820 \\
39,290 \\
58,110 \\
\end{array}$ & $\begin{array}{l}33 \\
66 \\
99 \\
\end{array}$ & $\begin{array}{l}0.570 \\
0.595\end{array}$ & 0,958 & 0,543 \\
\hline $\begin{array}{l}\text { Tiempo de permanencia } \\
\text { en el mismo centro }\end{array}$ & $\begin{array}{l}\text { Inter-grupos } \\
\text { Intra-grupos } \\
\text { Total } \\
\end{array}$ & $\begin{array}{l}25,809 \\
41,631 \\
67,440 \\
\end{array}$ & $\begin{array}{l}33 \\
66 \\
99 \\
\end{array}$ & $\begin{array}{l}0,782 \\
0,632\end{array}$ & 1,240 & 0,226 \\
\hline $\begin{array}{l}\text { Tipo de centro docente en } \\
\text { el que trabaja }\end{array}$ & $\begin{array}{l}\text { Inter-grupos } \\
\text { Intra-grupos } \\
\text { Total }\end{array}$ & $\begin{array}{r}8,411 \\
18,589 \\
27,000 \\
\end{array}$ & $\begin{array}{l}33 \\
66 \\
99 \\
\end{array}$ & $\begin{array}{l}0,256 \\
0,282\end{array}$ & 0,950 & 0,616 \\
\hline $\begin{array}{l}\text { Localización del centro } \\
\text { educativo }\end{array}$ & $\begin{array}{l}\text { Inter-grupos } \\
\text { Intra-grupos } \\
\text { Total }\end{array}$ & $\begin{array}{r}8,565 \\
16,345 \\
24,910 \\
\end{array}$ & $\begin{array}{l}33 \\
66 \\
99\end{array}$ & $\begin{array}{l}0,260 \\
0,248\end{array}$ & 1,048 & 0,425 \\
\hline
\end{tabular}

\section{CONCLUSIONES E IMPLiCACIONES}

La valoración de los análisis planteados conduce a una conclusión central, que -en opinión del profesorado consultado- el desarrollo de hábitos educativos en niños de 3 a 6 años favorece significativamente sus procesos de aprendizaje, así se han manifestado autores como Dahlberg y Asen (1994); Gútiez (1995); Moss y Penn (1996); no obstante se puede precisar esta conclusión en función de la incidencia de los diferentes hábitos trasmitidos por los profesores en los procesos de aprendizaje del alumnado de Educación Infantil.

Aunque la opinión es muy favorable, de modo generalizado, hacia el hecho de que todos los hábitos considerados favorecen el estudio de los alumnos, se manifiesta mayor unanimidad hacia la incidencia en el aprendizaje de hábitos conductuales y sociales, que, según el profesorado, se adquieren a través de actividades significativas y favorecen capacidades cognitivas, afectivas y relacionadas con la interacción social.

Son hábitos que suponen al profesorado el conocimiento de recursos, técnicas y capacidades complejas, a desplegar en los primeros compases en los que los niños inician el aprendizaje.

En el mismo sentido se plantea la necesidad de los hábitos relacionados con la apreciación y el cuidado del entorno y del propio cuerpo. No sólo porque favorecen una vida saludable y respetuosa con el medio ambiente, sino porque orientan a los 

DE EDUCACIÓN INFANTIL

niños hacia la indagación y la práctica de hábitos morales, con lo que implica todo ello para favorecer el estudio, tanto por el desarrollo de capacidades cognitivas en el primer caso, como afectivas, en el segundo, concretamente en el ejercicio de la voluntad.

También se contempla en las opiniones manifestadas que, en esos primeros momentos de la etapa, los alumnos tienen dificultades para iniciarse en los procesos lectoescritores, en la medida que necesitan alcanzar cierto nivel de madurez en el desarrollo de determinadas funciones cerebrales. Estos hábitos son considerados de vital importancia, ya que los procesos lectoescritores, además de facilitar la incorporación del niño al mundo y facilitar su socialización, favorecen el desarrollo de funciones cerebrales y ayudan a construir y desarrollar su capacidad discursiva.

También los hábitos tecnológicos y deportivos son considerados de vital importancia en la infancia, ya que favorecen la posterior adquisición de información en el niño durante toda la vida, tanto para la búsqueda, selección y gestión de la misma o la adquisición de rutinas para la utilización futura de nuevas tecnologías.

En este sentido anteriormente definido, no solo se adquiere información rápidamente, sino que tenemos la posibilidad de aprender, elaborado conocimiento, uno de los aspectos fundamentales del socioconstructivista. El desarrollo de la condición física favorece la adquisición de hábitos de trabajo en equipo, cierta exigencia en la elaboración de sus trabajos y, cómo no, la adquisición de hábitos de vida saludable mediante el ejercicio frecuente, que constituye la base de una buena salud corporal, necesaria para desarrollar el resto de actividades humanas.

Del mismo modo, se pone de manifiesto la opinión docente para la adquisición apropiada de hábitos psicomotores. Los docentes pueden ayudarse de la psicomotricidad, a través de procesos que vinculan la actividad del cuerpo con la cognitiva, proponiendo nuevos entornos que favorecen el desarrollo integral de los escolares. Plantea el profesorado la importancia del cuerpo para expresar mediante el lenguaje corporal lo que los niños y niñas piensan, manifestando también sus vivencias cognitivas como afectivas. La coordinación de lo motor y lo cognitivo favorece la unidad e integridad del niño como ser humano.

Con un elevado nivel de aceptación por el profesorado, se consideran los hábitos de higiene y autonomía personal de gran significación para el desarrollo de la vida del alumnado de estas edades. La higiene, tanto en los aspectos físicos como mentales, es necesaria para que los niños y niñas estén en condiciones de integrar aprendizajes. Manifiestan los docentes acuerdo en que se deben generar escenarios favorables que promuevan el desarrollo de autonomía en el niño, a través de estrategias y condiciones que beneficien tanto su higiene personal como sus aprendizajes. No se puede olvidar la importancia que la autonomía personal adquiere para favorecer la autoestima de los niños y, consiguientemente, el desarrollo de su identidad personal. 
MARÍA MARTÍNEZ REDONDO, MIGUEL PÉREZ FERRA Y BEATRIZ SIERRA Y ARIZMENDIARRIETA INCIDENCIA DE LOS HÁBITOS EDUCATIVOS EN EL APRENDIZAJE DEL ALUMNADO DE EDUCACIÓN INFANTIL

El análisis exploratorio realizado en la investigación se orienta en la misma línea de lo que se ha referido líneas atrás, confirmando los estudios descriptivos.

Tan solo el hecho de que el profesorado sea hombre o mujer evidencia diferentes opiniones respecto a la importancia de los hábitos para adquirir aprendizajes, el resto de variables no plantean modos diferentes de opinar, atendiendo a las diversas opciones de respuesta que contemplan las variables descriptivas. Todo ello permite pensar que la consideración de la incidencia de los objetivos educativos en los procesos de aprendizaje es una idea tan consolidada en las creencias pedagógicas de los docentes que apenas plantea opciones de valor diferentes.

Las reflexiones anteriores implican que la Educación Infantil es de vital importancia para el desarrollo de los alumnos en sus capacidades afectivas, cognitivas, psicomotoras y de interacción con el medio, lo que supone la necesidad de un profesorado preparado, con capacidad para seleccionar y gestionar materiales, diversificar actividades y trabajar en equipo.

Los profesores consultados son conscientes de las dificultades y déficits que arrastran los alumnos cuando en esta etapa de la vida, en la que se desarrollan de modo rápido y progresivo capacidades, habilidades y destrezas, el profesorado no ha abordado los procesos formativos con competencia profesional. Es por ello razón fundamental que, a partir de análisis como el presente, se aborden estudios relativos a la evaluación de materiales y métodos de trabajo que faciliten el desarrollo de los hábitos considerados, a través de estudios de evaluación de programas, correlacionales y pretest-postest, sin excluir estudios basados en historias de vida de los profesores sobre su desempeño profesional y conformación de su identidad profesional, así como su incidencia en el desarrollo de hábitos educativos en la etapa que nos ocupa.

En definitiva, los datos aportados por el profesorado en esta colaboración atestiguan la significación que tienen los hábitos analizados en la formación de los niños y niñas de 3 a 6 años, especialmente en el desarrollo y consolidación de aprendizajes. El hecho de adquirir rutinas y costumbres relacionadas con el ámbito didáctico facilita la adquisición de nuevos conocimientos, predisponen receptivamente a la adquisición de las enseñanzas y desarrollo de experiencias que son imprescindibles para la formación del alumnado de la etapa descrita.

\section{REFERENCIAS BIBLIOGRÁFICAS}

Abbott, R.; Berninger, V. y Fayol, M. (2010). Longitudinal relationships of levels of language in writing and between writing and reading in grades 1 to 7. Journal of Educational Psychology, 102 (2), 281-298.

http://dx.doi.org/10.1037/a0019318.

Adell, J. y Castañeda, L. (2010). Los Entornos Personales de Aprendizaje (PLEs): una nueva manera de entender el aprendizaje. En R. Roig Vila y M. Fiorucci (Eds.). Claves para la investigación en innovación y calidad educativas. La integración de las Tecnologías de la Información y la Comunicación y la Interculturalidad en las aulas (pp. 19-30). Alcoy: Marfil-Roma TRE Universita degli Studi. 
MARÍA MARTÍNEZ REDONDO, MIGUEL PÉREZ FERRA Y BEATRIZ SIERRA Y ARIZMENDIARRIETA INCIDENCIA DE LOS HÁBITOS EDUCATIVOS EN EL APRENDIZAJE DEL ALUMNADO DE EDUCACIÓN INFANTII

Ayers, W. (2004). Teaching the personal and the political: Essays on hope and justice. Nueva York: Teachers College Press.

Barber, M. y Mourshed, M. (2008). Cómo hicieron los sistemas educativos con mejor desempeño del mundo para alcanzar sus objetivos. Santiago de Chile: McKinsey y Company.

Bohera, C.; Piñol, R.; Freixenet, A.; Seix, V.; Lilomós, A. y Sadurní, L. (2010). Taller de manualidades. Barcelona: Parramón.

Buceta, J. M. (2004). Estrategias psicológicas para entrenadores de deportistas jóvenes. Madrid: Dykinson.

Capllonch, M. (2005). Las tecnologías de la información y la comunicación en la Educación Física de Primaria. Estudio sobre sus posibilidades educativas. Tesis doctoral. Universidad de Barcelona.

Castañeda, E. (2010). Equidad social y educación inicial: un asunto de derechos. En J. C. Tedesco (Ed.). Educación y justicia: el sentido de la educación. XXV Semana Monográfica de la Educación: La educación en el horizonte 2020 (pp. 31-33). Madrid: Santillana.

Connolly, P. (2003). The development of young children's ethnic identities: Implications for early years practice. En C. Vincent (Ed.). Social justice, education and identity (pp. 166-184). Londres: Routledge Falmer.

Cruz, J. (1997). Factores motivacionales en el deporte infantil y asesoramiento psicológico a entrenadores y padres. En J. Cruz (Ed.). Psicología del deporte. Madrid: Síntesis, pp. $147-176$.

Dalhberg, G. y Asén, G. (1994). Evaluation and Regulation: a question of emprovement of the Committee of Inquiry into the Quality of the Educational Experience offered to 3 and 4 years old. London: Ssmo.

Defior, S. y Serrano, F. (2005). The initial development of spelling in Spanish: From global to analytical. Reading and Writing, 18, 81-98. http://dx.doi.org/10.1007/s11145-004-5893-1.

Delgado, M. A. (2007). Reflexiones acerca de la práctica deportiva en edad escolar: dilema entre la realidad y las propuestas de cambio. Actas VII Congreso Deporte y Escuela (16-48). Cuenca: Diputación de Cuenca.

Domínguez de Ramírez, R. y Shapiro, E. S. (2007). Cross-language relationship between Spanish and English oral reading fluency among Spanish-speaking English language learners in bilingual education classrooms. Psychology in the Schools, 44, 795-806. Descargado el día 12 de mayo de 2013.

http://dx.doi.org/10.1002/pits.20260.

Entwitsle, N. J. (2000). Approaches to studying and leves of understanding: The influences of teaching and assessment. En Smart (Ed.). Higher education: Handbook of theory and research, vol. 15 (pp. 156-218). New York: Agathon Press.

Estivill, E. y Domènech, M. (2008). Cuidem el medi ambient i reciclem. Barcelona: Ara Llibres.

Farrel, A.; Tayler, C. y Tennent, L. (2006). Building social capital in early chilhood education and care: an Australian study. En R. Parker-Rees y J. Willan (Eds.). Early years Education. Major themes in education (pp. 623-632). Londres: Routledge.

Fons, M. (1999). Leer y escribir para vivir. Barcelona: Graó.

Foro Internacional de ONG y Movimientos Sociales (1994). Construyendo el futuro. Tratados alternativos de Rio '92. Madrid: Ministerio de Obras Públicas, Transportes y Medio Ambiente. Dirección General de Política Ambiental.

Freire, H. (2011). Educar en verde. Ideas para acercar a niños y niñas a la naturaleza. Barcelona: Graó. 

INCIDENCIA DE LOS HÁBITOS EDUCATIVOS EN EL APRENDIZAJE DEL ALUMNADO DE EDUCACIÓN INFANTIL

Gallego, C. (marzo 2006). Los prerrequisitos lectores. Ponencia presentada en el Congreso Internacional de Lectoescritura. Morelia (México).

García, A. (2009). La naturaleza y los niños. Madrid: CEP S. L.

Generelo, M. (2010). Las nuevas tecnologías y su aplicación en educación física. V Congreso Internacional XXVI Nacional de Educación Física: Docencia, innovación e investigación física (pp. 172-181). Barcelona: Facultad de Formación del Profesorado-INEF Barcelona.

González, M. J.; Romero, J. F. y Blanca, M. J. (1995). Modelo causal sobre el aprendizaje de la lectura. Psicothema, 7 (2), 377-390.

Gutiérrez, M. (2000). Actividad Física, estilos de vida y calidad de vida. Revista de Educación Física, 77, 5-16.

Gutiérrez, M. (2003). Manual sobre valores en la educación física y el deporte. Barcelona: Paidós.

Gútiez, P. (1995). La educación infantil: modelos de atención a la infancia. Revista Complutense de Educación Infantil, 6 (1), 101-113.

Jiménez, J. y O'Shanahan, I. (2008). Enseñanza de la lectura: de la teoría y la investigación a la práctica educativa. Revista Iberoamericana de Educación, 45 (5), 2-22.

Labat, H.; Ecalle, J. y Magnan, A. (2010). Effect of two-mode trainings of letter knowledge: Across-sectional study with 3-and 5 year-old children. Psichologie Française, 55 (2), $113-127$. http://dx.doi.org/10.1016/j.psfr.2009.12.002.

Ley 14/1970, de 4 de agosto, General de Educación y Financiamiento de la Reforma Educativa. Boletín Oficial del Estado, n.. 187 (6 de agosto de 1970).

Ley Orgánica 1/1990, de 3 de octubre, de Ordenación General del Sistema Educativo (LoGSE). Boletín Oficial del Estado, n.․ 238 (4 de octubre de 1990).

Ley Orgánica 10/2002, de 23 de diciembre, de Calidad de la Educación (LOCE). Boletín Oficial del Estado, n.o 307 (24 de diciembre de 2002).

Ley Orgánica 2/2006, de 3 de mayo, de Educación (LOE). Boletín Oficial del Estado, n. 106 (4 de mayo de 2006).

McLoughlin, C. y Lee, M. J. W. (2007). Listen and learn: A systematic review of the evidence that podcasting supports learning in high education. En C. Montgomerie y J. Seale (Eds.). Proceedings of world conference on educational multimedia, hypermedia and telecommunications (pp. 1669-1677). Chesapeake: VA. AAC.

McLoughlin, C. y Lee, M. J. W. (2010). Personalized and self regulated learning in the Web 2.0 era: International exemplars of innovative pedagogy using social software. Australasian Journal of Educational Technology, 26 (1), 28-43.

Mendoza, R.; Sagrera, M. R. y Batista, J. M. (1994). Conductas de los escolares españoles relacionadas con la salud (1986-1990). Madrid. Consejo Superior de Investigaciones Científicas.

Monjas, R. (Coord.) (2006). La iniciación deportiva desde un modelo comprensivo. Buenos Aires: Miño y Dávila.

Moss, P. (1994). The early childhood league in Europe: Problems and possibilities in cross national comparisons of levels of provision. European. Early Childhood Education Research Journal, 2 (2), 19-32. http://dx.doi.org/10.1080/13502939485207571.

Moss, P. y Penn, H. (1996). Transforming nursery education. London: Paul Chapman Publishing. 
MARÍA MARTÍNEZ REDONDO, MIGUEL PÉREZ FERRA Y BEATRIZ SIERRA Y ARIZMENDIARRIETA INCIDENCIA DE LOS HÁBITOS EDUCATIVOS EN EL APRENDIZAJE DEL ALUMNADO DE EDUCACIÓN INFANTIL

Nemirovsky, M. (1999). Sobre la enseñanza del lenguaje escrito. Barcelona: Paidós. Otero, A. R. y Bruno, C. M. (2009). Taller de Educación ambiental. Madrid: CEP S. L. Perrenoud, P. (2012). Cuando la escuela pretende preparar para la vida. Barcelona: Graó.

Rius, M. D. (2003). La grafomotricidad como un proceso lingüístico. Madrid: Ministerio de Educación y Ciencia.

Rodrigo, M. J.; Maíquez, M. L.; García, M.; Mendoza, R.; Rubio, A.; Martínez, A. y Martín, J. C. (2004). Relaciones padres-hijos y estilos de vida en la adolescencia. Psicothema, 16 (2), 203-210.

Salomon, G. y Schrum, L. (2007). Web2.0: New tools, new schools. Washington DC: International Society for Technology in Education.

Sellés, P. y Martínez, T. (2008). Evaluación de los predictores y facilitadores de la lectura. Bordón, 60 (3), 113-129.

Sevillano, M. L. (2007). Investigar para innovar en enseñanza. Madrid: Pearson Prentice Hall.

Soriano, M. (Coord.) (2007). Velocilector. Un programa multicomponencial de intervención en dificultades lectoras. Versión experimental no publicada. Valencia: Universidad de Valencia.

Tedesco, J. C. (2010). Educación y justicia: el sentido de la educación. En XXV Semana Monográfica de la Educación: La educación en el horizonte 2020. Madrid: Santillana.

Torres, J. (1999). Dinámica de los esfuerzos en actividades de condición física y salud. Actas en CD-ROM de las I Jornadas Andaluzas sobre Actividad Física y salud. Granada.

Valjataga, T.; Pata, K. y Tammets, K. (2011). Considering students' perspective on personal and distributed learning environments. En M. J. M. Lee y C. McLoughlin (Eds.). Web 2.0-based e-Learning: applying social informatics for tertiary teaching (pp. 85-107). Hershey, PA: IGI Global.

Wanzek, J. y Vaughn, S. (2007). Research-based implications from extensive early reading interventions. School Psychology Review, 36, 541-561.

Zabalza, M. Á. (1996). Didáctica de la Educación Infantil. Madrid: Narcea.

\section{ANEXO N.․․ 1}

ESCALA DE INCIDENCIA DE LOS HÁBITOS EDUCATIVOS DEL CURRÍCULUM DE EDUCACIÓN INFANTIL EN EL PROCESO DE APRENDIZAJE DEL ALUMNADO DE 3 A 6 AÑOS

\begin{tabular}{|c|c|c|c|c|c|}
\hline N.o Ítems & Ítems & 4 & 3 & 2 & 1 \\
\hline 1 & $\begin{array}{l}\text { Fomento los hábitos de higiene personal tales como el cepillado de } \\
\text { dientes y el aseo personal. }\end{array}$ & & & & \\
\hline 2 & $\begin{array}{l}\text { Proporciono al alumnado la realización de actividades tales como } \\
\text { la representación espontánea de personajes, hechos y situaciones a } \\
\text { través de su propio cuerpo. }\end{array}$ & & & & \\
\hline 3 & $\begin{array}{l}\text { Favorezco el desarrollo de experiencias manipulativas, tales como } \\
\text { construcción de palabras, juegos de escritura, donde el alumnado } \\
\text { adquiere aprendizajes significativos. }\end{array}$ & & & & \\
\hline 4 & $\begin{array}{l}\text { Desarrollo actividades para fomentar hábitos de esfuerzo, constancia, } \\
\text { y el placer por el trabajo bien acabado. }\end{array}$ & & & & \\
\hline 5 & $\begin{array}{l}\text { Expreso la importancia de llevar a cabo una alimentación rica, sana } \\
\text { y equilibrada. }\end{array}$ & & & & \\
\hline
\end{tabular}


MARÍA MARTÍNEZ REDONDO, MIGUEL PÉREZ FERRA Y BEATRIZ SIERRA Y ARIZMENDIARRIETA INCIDENCIA DE LOS HÁBITOS EDUCATIVOS EN EL APRENDIZAJE DEL ALUMNADO DE EDUCACIÓN INFANTIL

\begin{tabular}{|c|c|c|c|c|}
\hline \multicolumn{5}{|c|}{$\begin{array}{l}\text { ESCALA DE INCIDENCIA DE LOS HÁBITOS EDUCATIVOS DEL CURRÍCULUM DE EDUCACIÓN INFANTIL } \\
\text { EN EL PROCESO DE APRENDIZAJE DEL ALUMNADO DE } 3 \text { A } 6 \text { AÑOS }\end{array}$} \\
\hline N.o Ítems & Ítems & \begin{tabular}{|l|l|l}
4 & 3 \\
\end{tabular} & 2 & 1 \\
\hline 6 & $\begin{array}{l}\text { Posibilito situaciones de aprendizaje en las que el alumnado pueda } \\
\text { exteriorizar sus propios sentimientos. }\end{array}$ & & & \\
\hline 7 & $\begin{array}{l}\text { Organizo actividades para que el alumnado ponga en práctica } \\
\text { acciones de colaboración, cooperación y ayuda entre iguales. }\end{array}$ & & & \\
\hline 8 & $\begin{array}{l}\text { Propongo actividades de reflexión y de intercambio, entre el } \\
\text { alumnado, de opiniones sobre la prevención de accidentes y los } \\
\text { hábitos de salud y cuidado. }\end{array}$ & & & \\
\hline 9 & $\begin{array}{l}\text { Fomento en el aula actividades de reflexión y de acción de cuidado } \\
\text { y respeto al medio ambiente. }\end{array}$ & & & \\
\hline 10 & $\begin{array}{l}\text { Propongo desde la propia aula actividades de reciclaje de } \\
\text { materiales. }\end{array}$ & & & \\
\hline 11 & $\begin{array}{l}\text { Planteo situaciones lúdicas para aprender a respetar las diversas } \\
\text { formas de opinión y pensamiento. }\end{array}$ & & & \\
\hline 12 & $\begin{array}{l}\text { Resalto la importancia de que todas las personas tienen los mismos } \\
\text { derechos y que las relaciones entre ellas deben estar basadas en el } \\
\text { bienestar mutuo. }\end{array}$ & & & \\
\hline 13 & $\begin{array}{l}\text { Contribuyo al uso crítico y adecuado de las nuevas tecnologías tan } \\
\text { incipientes en nuestra actual sociedad. }\end{array}$ & & & \\
\hline 14 & $\begin{array}{l}\text { Facilito tareas educativas tecnológicas para adquirir habilidades y } \\
\text { destrezas básicas. }\end{array}$ & & & \\
\hline 15 & Planifico escenarios donde se fomente el placer por la lectura. & & & \\
\hline 16 & Manifiesto la importancia de realizar producciones escritas. & & & \\
\hline 17 & $\begin{array}{l}\text { Participo activamente en la iniciación y posterior desarrollo del } \\
\text { proceso lector. }\end{array}$ & & & \\
\hline 18 & $\begin{array}{l}\text { Los docentes, como modelo a seguir, presentan una actitud de } \\
\text { escucha activa para crear intercambios conductuales apropiados } \\
\text { con su alumnado. }\end{array}$ & & & \\
\hline 19 & $\begin{array}{l}\text { Considero necesaria la utilización de las nuevas tecnologías como } \\
\text { un recurso útil y práctico para potenciar el proceso de enseñanza y } \\
\text { aprendizaje de hábitos adecuados a estas edades. }\end{array}$ & & & \\
\hline 20 & $\begin{array}{l}\text { Insisto en que el abuso de los alimentos no saludables a estas edades } \\
\text { repercutirá negativamente en el equilibrio físico del alumnado. }\end{array}$ & & & \\
\hline 21 & $\begin{array}{l}\text { Una adecuada práctica de hábitos deportivos favorece un buen } \\
\text { estado físico y evito peligros en los alumnos. }\end{array}$ & & & \\
\hline 22 & $\begin{array}{l}\text { Me preocupan los hábitos afianzados no deseados (mala } \\
\text { alimentación, insuficiente higiene corporal, distorsionados hábitos } \\
\text { de sueño-vigilia) como reto para modificarlos. }\end{array}$ & & & \\
\hline 23 & $\begin{array}{l}\text { Realizo actividades en las que mi alumnado es el protagonista del } \\
\text { reciclado de envases. }\end{array}$ & & & \\
\hline
\end{tabular}


MARÍA MARTÍNEZ REDONDO, MIGUEL PÉREZ FERRA Y BEATRIZ SIERRA Y ARIZMENDIARRIETA INCIDENCIA DE LOS HÁBITOS EDUCATIVOS EN EL APRENDIZAJE DEL ALUMNADO DE EDUCACIÓN INFANTIL

\begin{tabular}{|c|c|c|c|c|}
\hline \multicolumn{5}{|c|}{$\begin{array}{l}\text { ESCALA DE INCIDENCIA DE LOS HÁBITOS EDUCATIVOS DEL CURRÍCULUM DE EDUCACIÓN INFANTIL } \\
\text { EN EL PROCESO DE APRENDIZAJE DEL ALUMNADO DE } 3 \text { A } 6 \text { AÑOS }\end{array}$} \\
\hline N. ${ }^{\text {Ítems }}$ & Ítems & 4 & \begin{tabular}{|l|l|}
3 & 2 \\
\end{tabular} & 1 \\
\hline 24 & $\begin{array}{l}\text { Priorizo la escritura como un aspecto de especial relevancia a través } \\
\text { de cuentos, comparaciones entre letras-frases, copias del alumnado, } \\
\text { etc. }\end{array}$ & & & \\
\hline 25 & $\begin{array}{l}\text { A través del juego propongo hábitos sociales que representen las } \\
\text { buenas conductas a seguir y se inhiban las no adecuadas. }\end{array}$ & & & \\
\hline 26 & $\begin{array}{l}\text { Favorezco hábitos de trabajo tales como la realización de fichas bien } \\
\text { acabadas para que en el futuro se vean reflejadas en hábitos de } \\
\text { estudio. }\end{array}$ & & & \\
\hline 27 & $\begin{array}{l}\text { Estimulo el gusto por aprender haciéndoles responsables de sus } \\
\text { actos. }\end{array}$ & & & \\
\hline 28 & $\begin{array}{l}\text { Es imprescindible aplicar estrategias educativas para la fomentar la } \\
\text { lectura. }\end{array}$ & & & \\
\hline 29 & $\begin{array}{l}\text { Dedico todos los días una parte de la jornada escolar a la } \\
\text { escritura. }\end{array}$ & & & \\
\hline 30 & $\begin{array}{l}\text { Aprovecho cualquier situación escolar para hacer uso de la lectura, } \\
\text { tales como envases de los desayunos, etiquetas, rótulos de la calle, } \\
\text { etc. }\end{array}$ & & & \\
\hline 31 & $\begin{array}{l}\text { Las TIC ayudan al proceso de enseñanza, por lo atractivo del } \\
\text { aprendizaje con el ordenador, software educativos, webquest, etc. }\end{array}$ & & & \\
\hline 32 & $\begin{array}{l}\text { Favorezco el cuidado del medio ambiente del alumnado con mi } \\
\text { modo de actuar. }\end{array}$ & & & \\
\hline 33 & $\begin{array}{l}\text { Propongo actividades donde el alumnado desarrolle la atención } \\
\text { durante un tiempo determinado. }\end{array}$ & & & \\
\hline 34 & $\begin{array}{l}\text { Es necesaria la realización diaria de actividades deportivas para el } \\
\text { desarrollo integral de nuestros alumnos. }\end{array}$ & & & \\
\hline 35 & $\begin{array}{l}\text { La utilización de los programas y juegos educativos interactivos } \\
\text { favorece positivamente el proceso de aprendizaje de nuestro } \\
\text { alumnado }\end{array}$ & & & \\
\hline 36 & $\begin{array}{l}\text { Los niños disfrutan con la escritura de sus propias producciones } \\
\text { expresando sus pensamientos, nombres, gustos, etc. }\end{array}$ & & & \\
\hline 37 & $\begin{array}{l}\text { El cuidado del cuerpo debe iniciarse desde las edades más } \\
\text { tempranas. }\end{array}$ & & & \\
\hline 38 & $\begin{array}{l}\text { Me planteo el reciclaje de materiales como un reto para que los } \\
\text { niños lo adquieran y desarrollen en su vida cotidiana. }\end{array}$ & & & \\
\hline 39 & $\begin{array}{l}\text { Priorizo la lectura en mi práctica docente diaria como factor } \\
\text { imprescindible para el posterior desarrollo educativo del } \\
\text { alumnado. }\end{array}$ & & & \\
\hline 40 & $\begin{array}{l}\text { Despierto interés por afianzar hábitos deportivos tales el gusto por } \\
\text { el ejercicio físico, la no competitividad y el disfrute por ayudar a los } \\
\text { demás. }\end{array}$ & & & \\
\hline
\end{tabular}

